\title{
QUEEN'S
UNIVERSITY
BELFAST
}

\section{D printed microneedle patches using stereolithography (SLA) for intradermal insulin delivery}

Economidou, S. N., Pere, C. P. P., Reid, A., Uddin, M. J., Windmill, J. F. C., Lamprou, D., \& Douroumis, D. (2019). 3D printed microneedle patches using stereolithography (SLA) for intradermal insulin delivery. Materials Science and Engineering C: Materials for Biological Applications. https://doi.org/10.1016/j.msec.2019.04.063

Published in:

Materials Science and Engineering C: Materials for Biological Applications

Document Version:

Peer reviewed version

Queen's University Belfast - Research Portal:

Link to publication record in Queen's University Belfast Research Portal

\section{Publisher rights}

Copyright 2019 Elsevier.

This manuscript is distributed under a Creative Commons Attribution-NonCommercial-NoDerivs License

(https://creativecommons.org/licenses/by-nc-nd/4.0/), which permits distribution and reproduction for non-commercial purposes, provided the author and source are cited

\section{General rights}

Copyright for the publications made accessible via the Queen's University Belfast Research Portal is retained by the author(s) and / or other copyright owners and it is a condition of accessing these publications that users recognise and abide by the legal requirements associated with these rights.

Take down policy

The Research Portal is Queen's institutional repository that provides access to Queen's research output. Every effort has been made to ensure that content in the Research Portal does not infringe any person's rights, or applicable UK laws. If you discover content in the Research Portal that you believe breaches copyright or violates any law, please contact openaccess@qub.ac.uk. 


\section{3D printed microneedle patches using}

2 stereolithography (SLA) for intradermal insulin

3 delivery

4 AUTHOR NAMES

5 Sophia N. Economidou', Cristiane Patricia Pissinato Pere ${ }^{1}$, Andrew Reid², Md. Jasim Uddin ${ }^{3}$,

6 James F.C. Windmill ${ }^{2}$, Dimitrios A. Lamprou ${ }^{4 *}$, Dennis Douroumis ${ }^{1 *}$

7 AUTHOR ADDRESS

$8 \quad{ }^{1}$ Medway School of Pharmacy, University of Kent, Medway Campus, Central Avenue, Chatham

9 Maritime, Chatham, Kent ME4 4TB, United Kingdom

$10{ }^{2}$ Centre for Ultrasonic Engineering, Department of Electronic and Electrical Engineering,

11 University of Strathclyde, 204 George St, Glasgow, G1 1XW, Scotland, United Kingdom

$12{ }^{3}$ Department of Pharmacy, BRAC University, Bangladesh. Address: 41 Pacific Tower,

13 Mohakhali, Dhaka-1212, Bangladesh.

$14{ }^{4}$ School of Pharmacy, Queen's University Belfast, 97 Lisburn Road, Belfast, BT9 7BL, United

15 Kingdom

16 Keywords

$173 \mathrm{D}$ printing, microneedles, inkjet coating, insulin, $\mu \mathrm{CT}$ 
19 Abstract

20 3D printed microneedle arrays were fabricated using a biocompatible resin through

21 stereolithography (SLA) for transdermal insulin delivery. Microneedles were built by

22 polymerising consecutive layers of a photopolymer resin. Thin layers of insulin and sugar

23 alcohol or disaccharide carriers were formed on the needle surface by inkjet printing. The

24 optimization of the printing process resulted in superior skin penetration capacity of the

25 3D printed microneedles compared to metal arrays with minimum applied forces varying

26 within the range of 2 to 5 N. Micro-CT analysis showed strong adhesion of the coated films

27 on the microneedle surface even after penetration to the skin. In vivo animal trials revealed

28 fast insulin action with excellent hypoglycaemia control and lower glucose levels achieved

29 within $60 \mathrm{~min}$, combined with steady state plasma glucose over 4 h compared to

$30 \quad$ subcutaneous injections.

32 Introduction

33 Transdermal Drug Delivery (TDD), the ability to effectively convey drugs through the human

34 skin, is an appealing concept, aiming at surpassing the pitfalls of the traditional administration

35 routes. The broader adoption of the transdermal route, however, is hampered by restrictions that

36 stem from the nature of the skin barrier itself, especially by the stratum corneum. Microneedles

37 (MNs) are small devices that can pierce this outermost, most impermeable layer of the human

38 skin and successfully deliver active substances such as drugs, Ribonucleic acid (RNA),

39 Deoxyribonucleic acid (DNA), and vaccines straight into the dermal microcirculation [1-4] .

40 Due to their small size they leave skin nerves intact upon insertion [5], while they increase 
41 bioavailability since the drug does not pass through any metabolic systems [6]. The MN-

42 mediated drug delivery is realised through multiple strategies that employ solid, coated, hollow,

43 hydrogel-forming and soluble MNs [7].

44 Since their introduction over 20 years ago, MN systems have attracted significant attention for 45 their potential to replace traditional drug administration routes. In the field of diabetes type 1 and 46 some types of type 2, the vast majority of patients rely on subcutaneous needle injections for 47 insulin replacement, a treatment approach highly associated with reduced patient-compliance [8].

48 Pain, skin thickening due to recurring injections, needle phobia and insulin leakages on the skin

49 surface $[9,10]$ have motivated vigorous research on MN-based systems for transdermal insulin 50 delivery.

51 Recent advances encompass the use of moulding techniques for the development of insulin-

52 loaded dissolvable MN systems. Wang et al. introduced a bioinspired MN system consisting of

53 dissolvable cross-linked poly(vinyl alcohol) (PVA) gel, catalase and glucose oxidase (GOx) that

54 responds to high glucose conditions by releasing insulin to the circulation. In vivo tests showed

55 that the systems were effective in maintaining normal blood glucose levels [11]. In another 56 study, modified alginate and hyaluronate were combined into a dissolvable, insulin57 encapsulating MN system. The MN arrays demonstrated good mechanical properties and skin 58 penetration capability while clinical studies demonstrated that the MNs almost fully dissolved 59 into the skin. The released insulin achieved a sustained hypoglycaemic effect and good relative 60 bioavailability of insulin, compared with subcutaneous injections [12]. Similar results in terms of 61 mechanical properties, relative insulin bioavailability and pharmacological activity were also 62 obtained by a study that manufactured composite dissolvable MNs [13]. The forenamed studies 
63 demonstrate that $\mathrm{MN}$ can be a promising alternative to subcutaneous injections for insulin 64 therapy.

65 Nonetheless, there are several hampering parameters that need to be taken under consideration

66 for the development of transdermal insulin delivery systems. The first relates to the time needed

67 for the detection of the drug into the systemic circulation; dissolvable systems are highly

68 dependent on the dissolution rate of the materials encapsulating the drug and may not be suitable

69 for fast insulin administration. To circumvent that issue, Ross et al. developed a solid MNs-

70 based system, coated with insulin-containing formulations through inkjet printing [14]. The use

71 of this technology permitted the accurate deposition of uniform and homogeneous coatings, with

72 high reproducibility. The implementation of inkjet printing for the development of thin layers on

73 the microneedle surface resulted in rapid insulin release within the first $20 \mathrm{~min}$.

74 Another pitfall stems from the use of moulding techniques, that involve a series of multiple,

75 often time-consuming steps. It is evident, that the upscaling of such processes can be

76 challenging. Furthermore, there is a lack of clinical data related to cytotoxicity of materials used

77 for moulded microneedles which actually limits their applications. Another important

78 disadvantage is the limited drug loading in polymeric microneedles without affecting their

79 mechanical properties and piercing capacity. In order to circumvent this issue several authors

80 proposed the use of large patches which in turn results in difficulties to apply the arrays in a

81 uniform manner and subsequently in dose variation of the administrated substances. Recently,

82 efforts have been made on the integration of the revolutionary technology of $3 \mathrm{D}$ printing as a

83 manufacturing method for MN-based systems. 3D printing or Additive Manufacturing (AM) is a

84 family of technologies that implement layer-by-layer processes to fabricate physical models,

85 based on a Computer Aided Design (CAD) model. 3D printing permits the fabrication of high 
86 degrees of complexity with great reproducibility, in a fast and cost-effective fashion [15-18]. In

87 the field of transdermal drug delivery systems, the use of photopolymerization-based techniques

88 such as Stereolithography (SLA), Digital Light Processing (DLP) and Two-Photon-

89 Polymerization (2PP) for the development of MNs has been reported [19-22]. Gittard et al.

90 fabricated MNs of various geometries for wound healing applications using a DLP system. The

91 MNs were then coated with silver and zinc oxide thin films by pulse laser deposition and their

92 antimicrobial character was verified [23]. In another study, drug-loaded MNs were developed

93 when a skin anticancer drug was incorporated into the photo-sensitive polymer blend prior to

94 photopolymerization through a micro-stereolithographic (DLP) apparatus [24].

95 In this study, 3D printed $\mathrm{MN}$ arrays featuring two different $\mathrm{MN}$ designs, pyramid and spear,

96 were developed employing a commercial SLA printer and a biocompatible Class 1 polymer. The

97 3D printed arrays were subsequently coated with insulin-sugar films using inkjet printing.

98 Mannitol, trehalose and xylitol were used as insulin carriers to preserve insulin activity prior to

99 the deposition of active films on the MNs surface. In vitro and in vivo studies demonstrated rapid

100 insulin release from the coated MN systems. The usage of SLA for 3D printing of microneedle

101 arrays is anticipated to overcome the existing disadvantages of conventional techniques by

102 providing high precision, rapid fabrication, reduced processing steps and freedom to print a wide

103 range of shapes.

104 Materials and methods

105 Materials

106 The insulin employed in this study was bovine and was procured in a $10 \mathrm{mg} \mathrm{mL}^{-1}$ solution from 107 Sigma-Aldrich (Gillingham, UK). Xylitol (Xylisorb ${ }^{\circledR}$ 90) and mannitol (Pearlitol $®$ ) were 
108 donated by Roquette Freres (France) while trehalose dihydrate was bought from Sigma-Aldrich

109 (Gillingham, UK). The resin used to fabricate the MNs was the biocompatible Class I resin,

110 Dental SG, by Formlabs. Streptozocin ( $\geq 75 \% \alpha$-anomer basis, $\geq 98 \%)$ and citric acid were both

111 purchased from Merck Chemical Co. (Darmstadt, Germany). All solvents were of analytical 112 grades.

113

114 Additive manufacturing of microneedles

115 The MN arrays were designed using an engineering software (SolidWorks, Dassault Systems) as

116 patches of $15 \times 15 \times 1 \mathrm{~mm}$. The patches featured two different needle shapes, a pyramid and a flat

117 spear shaped that geometrically resembled the shape of metallic MNs that has been studied

118 elsewhere [16]. This design, named 'spear' in the framework of this study, had base dimensions

119 of $0.08 \times 1 \mathrm{~mm}$, while the dimensions of the base for the pyramid MN were $1 \mathrm{x} 1 \mathrm{~mm}$. The length

120 of all MNs was $1 \mathrm{~mm}$ and all patches had a 6x8 needle layout, yielding $48 \mathrm{MNs}$ per patch. The

121 arrays were 3D printed using the Form 2 SLA printer by Formlabs with high resolution

122 capabilities (25 and 140 microns for $\mathrm{z}$ and $\mathrm{x}$ axes, respectively). After fabrication, the arrays

123 were washed in isopropyl alcohol bath to remove unpolymerized resin residues and then cured

124 for 60 min at $40{ }^{\circ} \mathrm{C}$ under UV radiation using the MeccatroniCore BB Cure Dental station.

125 Coating of microneedles through inkjet printing

126 An inkjet printer was employed (NanoPlotter II, Gesim, Germany) to print thin insulin-sugar

127 films on the surface of the 3D printed MNs. The inkjet printer forms the drug-containing films

128 depositing multiple layers of insulin - sugar droplets on each microneedle using a piezo-driven

129 dispenser (PicPip 300). In each coating cycle (layer), the dispenser jetted 2 droplets of 
130 formulation in 10 spots along each needle's longitudinal axis. A total of 92 coating cycles

131 resulted in a $10 \mathrm{UI}(350 \mu \mathrm{g})$ of insulin per array. The coated arrays were then incubated at room

132 temperature for 24 hours to allow the evaporation of the solvent (de-ionised water) and the

133 formation of uniform films. For the purposes of this study three coating formulations were used,

134 consisting of insulin:xylitol (5:1 wt/wt), insulin: mannitol (5:1 wt/wt) and insulin:trehalose (5:1

$135 \mathrm{wt} / \mathrm{wt}$ ) as $2 \%$ solid content. Prior to the coating process, the arrays were mounted on a metal stub

136 at $45^{\circ}$ relative to the dispenser, while its tip $(50 \mu \mathrm{m})$ was placed close to the $\mathrm{MN}$ surface to avoid

137 losses of material.

138 Scanning electron microscopy (SEM)

139 The coated MN arrays were mounted onto aluminium stubs using a double-sided carbon

140 adhesive tape (Agar Scientific, UK). Each coated MN array was examined by SEM (Hitachi SU

1418030 , Japan) using a low accelerating voltage (1.0kV). A low accelerating voltage was used to

142 avoid electrical charges on the MNs. The images of coated MNs were captured digitally from a

143 fixed working distance (11.6 mm) using different magnifications (e.g. 30, 80, 110 or $120 \mathrm{x}$ ).

\section{X-Ray Computer Micro Tomography}

145 X-Ray Micro Computer Tomography $(\mu \mathrm{CT})$ scans were performed on coated 3D printed

146 pyramid MN. The equipment employed was a Bruker Skyscan 1172, with an SHT 11 Megapixel

147 camera and a Hamamatsu $80 \mathrm{kV}(100 \mu \mathrm{A})$ source. The samples comprised of 3D printed pyramid

148 MN coated with the three insulin/sugar formulations; Sample A: insulin:xylitol (5:1 wt/wt),

149 Sample B: insulin: mannitol (5:1 wt/wt) and Sample C: insulin:trehalose $(5: 1 \mathrm{wt} / \mathrm{wt})$. After the

150 scans of the coated arrays were performed, the arrays were inserted in 8-ply strips of parafilm,

151 applying a force of $5 \mathrm{~N}$, to examine the performance of the coating during piercing and to 
152 investigate whether any coating material will remain on the parafilm surface, causing drug

153 losses. Moreover, the penetration depth was measured. The samples were mounted vertically on

154 a portion of dental wax and positioned $259.4 \mathrm{~mm}$ from the source. No filter was applied to the X-

155 Ray source and a voltage of $80 \mathrm{kV}$ was applied for an exposure time of 1,050 ms. The images

156 generated were $2,664 \times 4,000$ pixels with a resolution of $6.75 \mu \mathrm{m}$ per pixel.

157 A total of 962 images were taken in $0.2^{\circ}$ steps around one hemisphere of the sample with the

158 average of 4 frames taken at each rotation step. The images were collected and a volumetric

159 reconstruction of the sample generated by Bruker's CTvol software. The threshold for this

160 attenuation signal was set manually to eliminate speckle around the sample, and then further

161 cleaned with a thresholding mask using Bruker's CTAn software. The images produced by the

$162 \mu \mathrm{CT}$ are based on the level of attenuation though the sample, which is dependent on the

163 thickness of the material and its absorption coefficient. Here, it is assumed that the absorption

164 coefficient is linearly proportional to the density of the material and the resulting densities

165 expressed in Hounsfield Units (HU), with -1000 being the density of air and 0 being the density

166 of water.

167 Circular Dichroism (CD)

168 Insulin solution and the respective solutions of the insulin-sugar films were diluted to $1.0 \mathrm{mg}$ $169 \mathrm{~mL}^{-1}$ in deionised water and the spectra were recorded at $20^{\circ} \mathrm{C}$ between 190 and $260 \mathrm{~nm}$ by $\mathrm{CD}$ 170 (Chirascan, Applied Photophysics, UK) using a $0.1 \mathrm{~mm}$ polarization certified quartz cell 171 (Hellma). Spectra were recorded using a step size of $1 \mathrm{~nm}$, a bandwidth of $1 \mathrm{~nm}$ and an 172 acquisition time of 1 sec. Four scans were recorded for each sample, averaged and a 173 corresponding spectrum of water was subtracted from each spectrum. For estimation of the 
174 secondary structural composition of insulin, the CD spectra were evaluated using the CD SSTR

175 method [25].

176 Raman Spectroscopy

177 The films and their respective components were analysed using Raman microscopy (Jobin Yvon

178 LabRam I) with a laser of $532 \mathrm{~nm}$ wavelength coupled with an optical microscope with 50x

179 objective.

180 Penetration studies through porcine skin

181 The effect of the MN geometry on the force required to pierce the skin has been documented

182 [26]. In this study, to determine the effect of needle shape on the force required for skin

183 penetration, piercing tests using porcine skin were conducted. Identical piercing tests were

184 carried out using metallic MN arrays that have been studied and are described in literature [16],

185 to maintain a frame of reference with the respective studies. A texture analyser was employed,

186 and the MN array was mounted on the moving probe using double-sided adhesive tape. Prior to

187 testing, the porcine skin samples were placed in waxed petri dishes. Continuous force and

188 displacement measurements were recorded to identify the point of needle insertion. The speed of

189 the moving probe was $0.01 \mathrm{~mm} \mathrm{~s}^{-1}$.

190 Axial force mechanical testing of MNs

191 To evaluate the mechanical behaviour of the 3D printed microneedles, fracture testing under

192 axial loading was performed. The arrays were fixed onto a metal plate and were pressed against a

193 flat metal block attached to the moving head of a Tinius Olsen testing machine, until a pre-set

194 displacement of $500 \mu \mathrm{m}$ (height/2) was reached. Continuous force and displacement 
195 measurements were recorded to identify the point of needle failure. The speed of the moving

196 probe was $1 \mathrm{~mm} / \mathrm{s}$ and the experiments were replicated 5 times for each design.

197 Preparation of porcine skin for in vitro release of insulin

198 The release of insulin from the coated MNs through abdominal porcine skin was studied using 199 Franz diffusion cells (PermeGear, Inc., PA, USA). The full thickness abdominal porcine skin 200 was collected from a local slaughterhouse (Forge Farm Ltd, Kent, UK) and was then shaved 201 using a razor blade. The fatty tissue below the abdominal area of porcine skin was removed with 202 scalpel and then pinned onto polystyrene block and wiped with $70 \%$ ethanol. The skin was then 203 cut by applying the dermatome at an angle of $\pm 45^{\circ}$ (Padgett dermatome, Integra LifeTMSciences 204 Corporation USA). The thickness of the skin was measured by using a calliper and the tissue 205 disks of the required dimensions were cut for the Franz diffusion cells using a scalper. The skin 206 tissue $(1.0 \pm 0.1 \mathrm{~mm}$ thick) was placed onto filter paper soaked in a small amount of saline 207 phosphate buffer ( $\mathrm{pH} 7.4)$ for $2 \mathrm{~h}$.

208 In vitro release of insulin through porcine skin

209 A total diffusion area of $1.1 \mathrm{~cm}^{2}$ was used to assess the insulin release. The MN arrays were 210 inserted into the abdominal porcine skin samples for $30 \mathrm{~s}$, via manual finger pressure. The 211 sample was then mounted onto the donor compartment of a Franz diffusion cell. The temperature 212 of the Franz cells was maintained at $37^{\circ} \mathrm{C}$ using an automated water bath (Thermo Fisher 213 Scientific, Newington, USA). Sample fractions $\left(6-6.5 \mathrm{~mL} \mathrm{~h}^{-1}\right)$ were collected using an auto214 sampler (FC 204 fraction collector, Gilson, USA) attached to the Franz diffusion cells system. 215 Statistical analysis for the drug release was performed by using a Mann-Whitney nonparametric 
216 test and t-test analysis for the in vivo studies (InStat, GraphPad Software Inc., San Diego, CA,

217 USA), where samples were considered as statistically significant at $\mathrm{p}<0.05$.

218 High-Performance Liquid Chromatography (HPLC)

219 The amount of insulin collected from the receptor fluid was determined by HPLC (Agilent

220 Technologies, 1200 series, Cheshire, UK) equipped with a Phenomenex Jupiter 5u c18 $300 \AA$,

221 LC Column $(250 \times 4.60 \mathrm{~mm}$, particle size $5 \mu \mathrm{m}$, Macclesfield, UK). The mobile phase consisted

222 of water with $0.1 \%$ Trifluoroacetic Acid (TFA) and acetonitrile with $0.1 \%$ TFA (66:34v/v), with

223 a $1 \mathrm{~mL} \mathrm{~min}{ }^{-1}$ flow rate. The column was equilibrated at $35^{\circ} \mathrm{C}$, the injection volume was $20 \mu \mathrm{L}$

224 and the eluent was analysed with a UV detector at $214 \mathrm{~nm}$. The results were integrated using

225 Chemstation ${ }^{\circledR}$ software and the samples analysed in triplicates.

226 In vivo release in diabetic mice

227 Prior to the induction of diabetes, Swiss albino female mice $(120 \pm 10 \mathrm{~g})$ were allowed free

228 access to solid bottom cages with controlled diet and water for 3 days. Mice were subcutaneously

229 injected on the flank with streptozotocin $\left(70 \mathrm{mg} \mathrm{kg}^{-1}\right)$ in citric acid buffer ( $\left.\mathrm{pH} 4.5\right)$ to produce a

230 diabetic animal model. To confirm the induction of diabetes, the fasting blood glucose level was

231 measured at scheduled times using a one-touch glucometer (ACCU-CheckVR Active, Roche,

232 Germany). After one week, mice with blood glucose exceeding $300 \mathrm{mg} / \mathrm{dl}$ were considered as

233 diabetic. The diabetic animals were anesthetised and shaved carefully using an electric razor

234 (Panasonic, USA) 24 hours prior to the experiments. Furthermore, the diabetic mice were fasted

235 for 12 hours before the beginning of the study, receiving only water and libitum. The mice were

236 randomly divided into three groups ( $\mathrm{n}=3$ for each group): (1) untreated group as negative

237 control; (2) subcutaneous injection (SC; 0.2 IU/animal) as positive control; (3) 3D printed MN 
238 (0.2 IU/array). The 3D printed MN arrays were applied onto the dorsal skin of the animals using 239 adhesive tape (3M, USA) to prevent any dislodgement during therapy. After 2 hours, the 3D 240 printed MN patches were removed. For all groups, blood samples were collected from the 241 jugular vein at $0,1,2,3$ and 4 hours after the insulin administration and the blood glucose level 242 was measured using the glucometer mentioned. Plasma insulin concentrations were measured via 243 an insulin-EIA Test kit (Arbor Assays, MI, USA). The treatment strategy is described in Table 1.

244 All animal experiments throughout this study were approved by the Research Ethics Committee 245 (reference number 0003/17, Department of Pharmacy, Southern University Bangladesh) and 246 conducted according to the Southern University Bangladesh policy for the protection of

247 Vertebrate Animals used for Experimental and Other Scientific Purposes, with implementation 248 of the principle of the 3Rs (replacement, reduction, refinement). No skin reactions to MNs 249 occurred.

250 Pharmacodynamic and pharmacokinetic profile of insulin-coated 3D printed MNs

251 The minimum glucose level (Cmin) and the time point of minimum glucose level (Tmin) were 252 calculated from the plasma glucose level versus time curve. The relative pharmacological 253 availability (RPA) was calculated using equation 1.

$254 \operatorname{RPA}(\%)=(\mathrm{AAC} 3 \mathrm{DMN} \times \operatorname{dosesc}) /(\mathrm{AACsc} \times \operatorname{dose} 3 \mathrm{DMN}) \times 100$

255 Where AAC3DMN indicates the area above the curve after the application of the insulin-coated 256 3D printed MNs, and AACsc shows the area above the curve after the subcutaneous injection of 257 insulin. 
The maximum plasma insulin concentration (Cmax) and the time point of maximum plasma

259 insulin concentration (Tmax) were calculated from the plasma insulin concentration $(\mu \mathrm{IU} / \mathrm{ml})$

260 versus time curve. The relative bioavailability (RBA) was determined using equation 2.

$261 \quad \operatorname{RBA}(\%)=(\mathrm{AUC} 3 \mathrm{DMN} \times \operatorname{dosesc}) /(\mathrm{AUCsc} \times \operatorname{dose} 3 \mathrm{DMN}) \times 100$

262 Where AUC3DMN indicates the area under the curve after the application of the insulin-coated

263 3D printed MNs, and AUCsc shows the area under the curve after the subcutaneous injection of 264 insulin.

265 Results and Discussion

266 Additive Manufacturing and printability of microneedles

267 MN arrays featuring pyramid and spear needles were 3D printed using a commercial SLA printer

268 based on digital CAD designs developed via appropriate engineering software. The polymer 269 employed was a photo-sensitive Class I resin which has been FDA approved. All arrays were 270 washed and subsequently cured under UV radiation in a controlled temperature environment to 271 improve the material's mechanical performance.

272 The capability of 3D printing technology to manufacture complex structures reproducibly and 273 accurately in a one-step-fashion, was exploited in this work to build different designs of MN 274 arrays. Although the degree of complexity that can be achieved through 3D printing is often not 275 achievable through many conventional techniques of $\mathrm{MN}$ manufacturing, the technology is 276 hampered by restrictions in terms of resolution that can affect the formation of sharp MN tips. 277 Conventional low-budget SLA printers have a maximum resolution of 100 microns that is 278 governed by the size of the laser focal point and restricts the minimum size of MN tip that can be 
279 formed. The MNs designed in the framework of this study featured a tip of 100 microns by 280 design and their penetration capability through porcine skin was tested to verify that they will 281 successfully and painlessly pierce the skin.

282 The printability of MNs was further improved when printing-in-an-angle was implemented, 283 leading to finer, sharper MN tips. An innate characteristic of the SLA technology is the 284 interdependence between the print quality and the cross-sectional area in the z-axis; the smaller 285 the z-axis cross-sectional area, the better the quality. This stems from the peel-off function of the 286 printing process, according to which, after the completion of each layer, a wiper slides and peels 287 the structure off the bottom of the resin tank. Larger z-axis cross sectional areas lead to greater 288 forces applied by the wiper, which can deform the printed structures. Orienting the part to 289 minimise the contact area of the structure to the resin tank avoids the possible distortions during 290 the peel-off process and leads to better print quality.

291 SLA parts are considered mechanically isotropic which ensures the mechanical properties of the 292 arrays are not affected by the angle of printing. In addition, an influential factor of the 293 mechanical performance of the MN arrays is the selection of post-process curing parameters 294 (time and temperature). Further research is required to determine the effect of those factors on 295 the overall mechanical and piercing behaviour of the MN arrays. In this work, the MNs were 296 cured in a UV chamber for $60 \mathrm{~min}$ in temperature of $40{ }^{\circ} \mathrm{C}$. It is demonstrated in this study that 297 those parameters yielded systems that successfully pierced through porcine skin requiring small 298 forces, with no needle failure occurring.

299 As discussed above due to technical limitations of the existing MN manufacturing techniques 300 (e.g. moulding, lithography) such as limited drug loading, dose consistency and scalability issues 
301 there are no commercialized products. Polymeric MNs are fabricated using moulding approaches

302 while metal MNs implement dip - coating techniques which renders both approaches impractical

303 for large scale manufacturing. In contrast scale-up of SLA printed MNs is directly related to the

304 usage of large volume printers or the in-line arrangement of existing printers. We envisage that

305 the implementation of SLA printed MNs will open new horizons for transdermal drug delivery

306 due to the low cost of the printers, printing inks and fast fabrication times.

307 SEM analysis demonstrated that through the use of the SLA technology, uniform and 308 reproducible arrays were developed (Fig. 1a,b). In Fig. 1, the high consistency and 309 reproducibility of the MN layers is depicted, and the formation of sharp tips is demonstrated. It is 310 evident, that the high-resolution capabilities of the printer allowed the parallel fabrication of 311 identical and reproducible arrays with characteristics that favour the skin insertion.

312 Coating of microneedles through inkjet printing

313 Insulin and sugar alcohol coatings were formed on the surface of the $3 \mathrm{D}$ printed MNs using

314 inkjet printing and a piezoelectric dispenser. A similar process was developed in earlier studies

315 for coating metallic MNs [16,27], where the applied voltage $(\mathrm{mV})$ and pulse duration (ms) were

316 tuned to achieve the production of droplets of $300 \mathrm{pL}$ volume with particle size of 100-110 $\mu \mathrm{m}$.

317 Fig. 1c,d illustrates the uniformity and reproducibility of the coatings on the MN surface without 318 any losses of material in the form of satellite droplets on the substrate. Moreover, it is 319 demonstrated that the consecutive jetting cycles produced drug-containing films that are smooth 320 and level in comparison to other techniques such as dip coating that may yield voluminous and 321 inconsistent coatings. This smooth morphology of the films prevents the losses of drug during 322 MN insertion that occur when bulky coatings remain on the skin surface. 
323 The drug carriers selected were two alcohol sugars (xylitol, mannitol) and a disaccharide

324 (trehalose). Those excipients have been reported to favour the immediate coating dissolution in

325 the skin and to enhance insulin stability in solid state [28-30] .

326 X-Ray Computer Micro Tomography

327 The coated 3D printed pyramid MN arrays were scanned using the Bruker Skyscan 1172 and an 328 overview of the array is presented in Fig. 2a. For sample A, an average needle base area of 1.095

$329 \mathrm{~mm}^{2}$ and an average needle height of $1.034 \mathrm{~mm}$ were measured. For samples B and C, the 330 average needle base areas were measured as $1.065 \mathrm{~mm}^{2}$ and $1.091 \mathrm{~mm}^{2}$ and the average needle 331 heights as $1.040 \mathrm{~mm}$ and $1.038 \mathrm{~mm}$, respectively. The average interspacing of the pyramids 332 between the centre points was $1.842 \mathrm{~mm}, 1.865 \mathrm{~mm}$, and $1.864 \mathrm{~mm}$ between columns, and 1.788 $333 \mathrm{~mm}, 1.810 \mathrm{~mm}$, and $1.796 \mathrm{~mm}$ between rows for samples A, B and C respectively. Scans taken 334 from the left-hand side of the arrays illustrate the thin coating films fabricated through inkjet 335 printing, in comparison with respective ones taken from the back side of the array (Fig. 2b,c).

336 The relative density of the MNs relative to the control sample (uncoated 3D printed pyramid 337 MNs) showed an increase of approximately $200 \mathrm{HU}$ between all coated samples and the control 338 (Fig. 3a,b). Profile lines across a row of MNs revealed a coffee-ring effect in the density of the 339 coating material deposition. While denser material was distributed randomly within each of the 340 MNs, a fringe layer of 10-15 $\mu \mathrm{m}$ was apparent with the effect being most pronounced in the 341 insulin:xylitol coated sample (Fig. 3c).

342 Penetration experiments in 8-ply strips of parafilm were performed applying a $5 \mathrm{~N}$ force (Fig. 4).

343 The penetration depth was measured as $559 \mu \mathrm{m}, 662 \mu \mathrm{m}$ and $650 \mu \mathrm{m}$ for samples A, B and C, 
344 respectively. The $\mu \mathrm{CT}$ scans illustrate that the coating stays on the MN surface throughout the

345 piercing process and there is no material remaining on the parafilm surface.

346 Circular Dichroism

347 Circular dichroism (CD) spectroscopy is a reliable technique for the evaluation of the secondary 348 structure of proteins in a solution. The influence of the two polyols and the disaccharide on 349 insulin molecule as well as their interactions were studied using CD and the estimation of insulin 350 secondary structure was performed by CDSSTR method [31,32].

351 The far-UV CD spectra of insulin and insulin-sugar films (Fig. 5a) were found to be coincident 352 with the one of standard insulin solution, showing double minima around 210 and $222 \mathrm{~nm}$ which 353 are typical of predominant $\alpha$-helix structure proteins as already reported elsewhere [28,33-35].

354 However, a slight decrease in Molar ellipticity is noted when insulin solution is dried which is 355 also supported by the decrease of the estimated percentage of $\alpha$-helix and increase of the $\beta$-sheet 356 content. Such behaviour may be indicative of the unfolding tendency of insulin during 357 dehydration [36]. Interestingly, once the sugars were added, all the respective insulin-sugar films 358 spectra showed higher Molar intensities than the insulin film alone, indicating an increase in the $359 \alpha$-helix content. The protective property of those sugars can be explained by the water 360 replacement mechanism which proposes that sugars may maintain the three-dimensional 361 structure of proteins by hydrogen-bonding with them $[37,38]$

362 Among the tested sugars, xylitol presented the best capability to maintain insulin in its native 363 secondary structure with even higher amounts of $\alpha$-helix content. The reason for this still remains 364 unclear and further research is needed.

365 Raman Spectroscopy 
366 In this work, the Raman spectrum of native insulin shows a strong peak at $1661 \mathrm{~cm}^{-1}$ due to the 367 amide I mode of $\alpha$-helix structure and a shoulder at $1682 \mathrm{~cm}^{-1}$ which is attributed to random coil 368 form as previously reported by $\mathrm{Yu}$ et al. [40]. Distinctive peaks of sugars were not found in

369 Raman mainly because insulin was 5 times more concentrated than the sugars in the films. 370 Likewise, the amorphous nature of the dried formulation (XRD analysis - data not shown) is 371 unlikely to afford a strong Raman signal.

372 Overall, insulin-sugar formulations showed similar Raman spectra to the native insulin (Fig. 5b).

373 Nonetheless, a slight shift in amide I band position can be seen for all formulations. Amide I

374 band of insulin-xylitol and insulin-mannitol formulations was shifted towards greater frequency,

$3751663 \mathrm{~cm}^{-1}$ and $1662 \mathrm{~cm}^{-1}$, respectively, while insulin-trehalose band was shifted to lower 376 frequency at $1658 \mathrm{~cm}^{-1}$. Those events were also reported by Carpenter and Crowe [37] and 377 Souillac et al. [41], who described that those changes might be due to the different effect of each 378 sugar on the vibrational spectra of insulin as well on the hydrogen bonding and couplings 379 between the adjacent peptide units.

380 Many researchers have investigated the protective properties of different sugars on polypeptides, 381 proteins and biomolecules [42-44]. It has been advocated that protein aggregation and 382 denaturation can be prevented by using carbohydrates as protectants. Protein protection by the 383 sugars in a dried system can be explained by the water replacement mechanism which suggest 384 the sugars may substitute water molecules around the biomolecules of proteins, maintaining its 385 three-dimensional structure by providing sites with hydrogen-bonding species $[37,45,46]$.

386 Zeng et al. studied the impact of relative humidity $(\mathrm{RH})$ on dehydration of insulin crystals and 387 they found the hydration water from insulin crystal can be gradually excluded when the RH is 
388 decreased. They used the high frequency region in Raman spectroscopy to access the band at $389 \sim 3450 \mathrm{~cm}^{-1}$ which is caused by both water and amino acid residues with $\mathrm{O}-\mathrm{H}$ groups. A 390 continuous dropping of the $\mathrm{O}-\mathrm{H}$ stretching band around $3450 \mathrm{~cm}^{-1}$ was observed while the RH 391 was reduced, indicating dehydration of the molecule [47].

392 From Fig. $5 \mathrm{~b}$ it can be seen that for all formulation the S-S vibration bands are located close to $393513 \mathrm{~cm}^{-1}$ suggesting that all disulphide bonds are in an adopted more stable gauche - gauche 394 gauche conformation $[47,48]$ as a result of the complete water removal during inkjet printing. 395 Tyr residues present Raman peaks at $642,828,852$, and $1174 \mathrm{~cm}^{-1}$, while the $1206 \mathrm{~cm}^{-1}$ peak is 396 related to both Tyr and Phe residues. Furthermore, the ratios of $\mathrm{I}_{852 / 1828}$ and $\mathrm{I}_{1174 / 11206}$ varied from $3970.91-1.02$ and $0.77-0.81$ respectively. These values are much lower compared to those 398 observed from Zeng et al. [47], for insulin crystals at very low RH (2\%). This phenomenon 399 suggests significant water loss of the coated formulation and stronger $\mathrm{H}$ - bonding interactions.

400 Vibrational modes in the area of $1100-1300 \mathrm{~cm}^{-1}$ have shown to be sensitive to the changes of 401 hydrogen bonds which involve the phenolic hydroxyl groups of Tyr residues and particularly the $402 v_{7 a}$ frequency. In Fig. $5 \mathrm{~b}$ the $v_{7 a}$ has a frequency of $1275 \mathrm{~cm}^{-1}$ which is a robust evidence that the 403 phenolic $\mathrm{OH}$ group of Tyr is strongly hydrogen bonded to a base atom [49].

404 Penetration studies through porcine skin

405 The 3D printed pyramid and spear shaped MN arrays were tested for their porcine skin 406 penetration capability using a texture analyser. Identical experiments were performed using 407 metallic MNs and the results were compared to the respective ones obtained from the 3D printed 408 MN experiments. All piercing tests were successful with no MN damage or failure. Throughout 409 each test, measurements of force and displacement were taken (Fig. 6a). 
410 All curves presented an initial linear segment (displacement $<0.3 \mathrm{~mm}$ ); after that, the slope was

411 changing constantly until a maximum force value was reached and a steep decrease of the force

412 was observed. This value is identified as the maximum force required for MN insertion [26]. The

413 non-linear behaviour of the force-displacement curve indicates that the process of $\mathrm{MN}$ insertion

414 to the skin is comprised of small penetrations where the MNs gradually tear the skin, before the

415 load reaches the maximum value that makes the insertion abrupt [50]. The maximum force

416 required for the MNs to successfully pierce the skin plays a crucial role when different MN

417 designs need to be compared. As presented in Fig. 6b, the pyramid MN required the least amount

418 of force to penetrate the porcine skin.

419 Axial force mechanical testing of MNs

420 The two studied MN designs were tested under compressive axial loading to determine the force

421 of microneedle fracture as a function of geometry. The force vs displacement measurements and

422 respective fracture strength values are presented in Fig. 7.

423 The two designs exhibited different mechanical behaviours during testing. For both designs, the 424 recorded force increased until the ultimate load was reached, and fracture occurred. For the spear 425 MNs, the point of fracture appears as a peak at approximately $175 \mathrm{~N}$, followed by a drop of the 426 recorded load; as the MNs were kept being pressed against the metal block after fracture, the 427 load was considerably decreased. On the contrary, the pyramid geometry showed a discontinuity 428 at approximately $457 \mathrm{~N}$ which is identified as the point of initial needle failure. Afterwards, the 429 load kept increasing as the microneedles kept being compressed. This difference in mechanical 430 behaviour is attributed to the different modes of needle failure. On the one hand, the spear MNs 431 fractured in the lateral direction, perpendicular to the loading axis, a finding that was confirmed 
432 by visual observation. This mode of failure was expected, due to the small thickness of the MNs

433 in that direction, which translates to minimisation of area, thus increased stress fields. On the

434 other hand, the pyramid MNs failed under pure compression, with the tip failing first and

435 additional, increasing force required for the compression of the remaining MN body. These

436 findings verify that both designs are safe for application since the fracture strengths of the arrays

437 are far greater than the respective forces needed for needle penetration through porcine skin.

438 They also confirm that the pyramid geometries present the best potential between the two studied

439 designs.

440 In vitro release of insulin through porcine skin

441 The in vitro insulin release studies from 3D printed pyramid and spear MNs were investigated

442 using porcine skin in Franz cells. The used carriers, mannitol, trehalose and xylitol not only

443 preserved insulin in its native form but also provided fast dissolution rates. As shown in Fig.

$4448 \mathrm{a}, \mathrm{b}$ for the pyramid designs approximately $80 \%$ of insulin was released in the first 2 min with

$44586-92 \%$ within 8 min. The rapid release profiles were obtained for all insulin carriers and no

446 statistical difference was observed (two-tail $\mathrm{p}=0.0021$ ).

447 The coating of each pyramid side resulted to higher surface area exposed for hydration and thus 448 faster hydration rates. In contrast, the spear 3D printed designs presented slightly slower insulin 449 release rates with $62-70 \%$ and $81-84 \%$ released within 2 min and $8 \mathrm{~min}$ respectively. Overall, 450 the rapid insulin release rates of the 3D printed MNs was attributed to the hydrophilic nature of 451 the three carriers and the thin coating layers $(10-15 \mu \mathrm{m})$ as shown from the $\mu \mathrm{CT}$ analysis.

452 In vivo transdermal delivery of insulin in diabetic mice 
453 Diabetes was successfully induced in mice after 7 days of streptozotocin administration. The 454 preliminary diabetes (hyperglycemia) was demonstrated as $340 \pm 10 \mathrm{mg} / \mathrm{dl}$. The diabetic mice 455 were divided into three groups: Untreated (negative control), subcutaneously (SC) injected 456 (positive control) and treated with the 3D printed insulin-coated MNs. Fig. 9 shows the 457 application process of the 3D printed MN arrays.

458 The dose 0.2 IU/array was selected in order to avoid hypoglycaemia in mice for $4 \mathrm{~h}$. The 459 comparative studies on different delivery strategies in plasma glucose levels are shown in Fig. 460 10a. Insulin-coated 3D printed MN arrays showed a remarkable steady state hypoglycaemia 461 effect (32.8\% from total value) in comparison to negative and positive control. After an hour, 462 subcutaneous injection (0.2 IU/injection) facilitated a rapid increase in insulin concentration in 463 blood and hence the decrease in plasma glucose level was approximately $30.1 \%$ from its primary 464 value. A comparable blood glucose regulation was observed between the SC group and the MN 465 group which was achieved within $1 \mathrm{~h}$ [51]. Interestingly, the 3D printed patch presented the same 466 rate to reach its lowest glucose level compared to the SC injection. In similar study 467 biodegradable MN patches Tmin of glucose levels was achieved within $2 \mathrm{~h}$ with administrated 468 doses of $5-10 \mathrm{IU}$ per patch [13]. The main reason for the faster glucose rates is that in Zhang et 469 al. (2018) insulin was encapsulated in moulded $\mathrm{MN}$ patches while for the 3D printed patches is 470 applied on the microneedle surface with very hydrophilic thin layers resulting in rapid insulin 471 release. Moreover, previous studies have shown that microneedle injection of insulin to human 472 diabetic subjects was chosen over hypodermic infusion and that pharmacokinetics were faster 473 when insulin was administered to the skin compared to the subcutaneous injections [52].

474 Although the plasma glucose level versus time profile was similar to previous findings [53-57] 475 the steady state plasma glucose level was maintained up to $4 \mathrm{~h}$ while the untreated group 
476 (negative control) remained unchanged (no hypoglycaemia) for the same period. These findings

477 suggest that insulin is being released from the 3D printed MNs to the mice blood stream via 478 passive diffusion to blood capillary.

479 Fig. 10b illustrates the plasma insulin concentration versus time where both SC and 3D printed 480 MN groups achieved the highest amount of plasma insulin concentration after $1 \mathrm{~h}$ of 481 administration. The control group did not show any detectable plasma insulin concentration. The

482 highest insulin level of the 3D printed MNs is slightly lower to the SC injection but no statistical 483 difference was observed. As shown in Fig. 10b after post - administration for $4 \mathrm{~h}$ the serum 484 insulin of 3D printed microneedles was higher to the SC injection.

485 Tables 2 and 3 represent the pharmacodynamic parameters of plasma glucose levels and the 486 pharmacokinetic parameters for plasma insulin concentrations, respectively. The RPA and RBA 487 for the insulin-coated 3D printed MNs group were both about $85-96 \%$. These results indicate that 488 insulin released from 3D printed MNs was almost completely absorbed from the skin into the 489 systemic circulation, and the pharmacological activity of the released insulin remained intact 490 after the delivery with the 3D printed MNs.

491 Conclusions

492 MN arrays of high quality and reproducibility, featuring spear and pyramid-shaped needle 493 geometries, were successfully fabricated by a biocompatible resin using stereolithography. The 494 3D printed polymeric MNs required low forces to penetrate porcine skin, in comparison with 495 metallic MNs. Uniform and accurate insulin-sugar thin layers were applied on the surface of the 496 MNs through inkjet printing, with no satellite droplets detected on the substrate. The insulin 497 integrity was found to be preserved by all carriers, namely the $\alpha$-helix and $\beta$-Sheet, with xylitol 
498 showing the optimum performance. In vivo animal trials demonstrated that 3D printed MNs

499 facilitate rapid low glucose levels with longer duration compared to SC injections. 
501 FIGURES
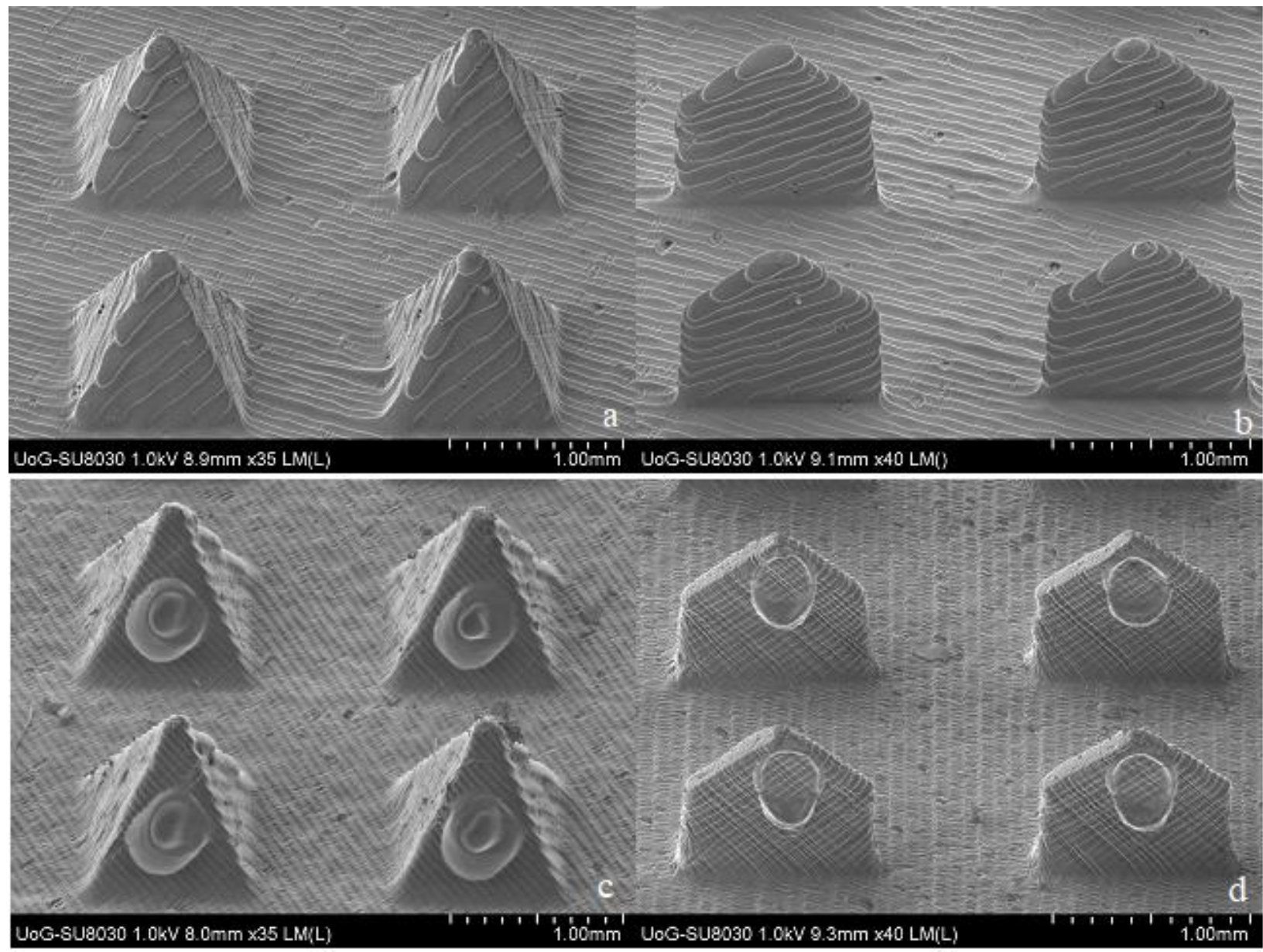

503 Figure 1. SEM images of the 3D printed MNs. (a) Uncoated pyramid; (b) uncoated spear; (c)

504 coated pyramid; (d) coated spear. The thin coating films on the MNs were created using an inkjet

505 printer. The formulations employed for the coatings contained insulin and a sugar used as a

506 carrier (xylitol, mannitol and trehalose) in a 5:1 ratio. Each MN patch was coated with 10 IU. All

507 insulin-carrier combinations formed coatings with similar morphology. The carrier used for

508 captions (c) and (d) is xylitol. 

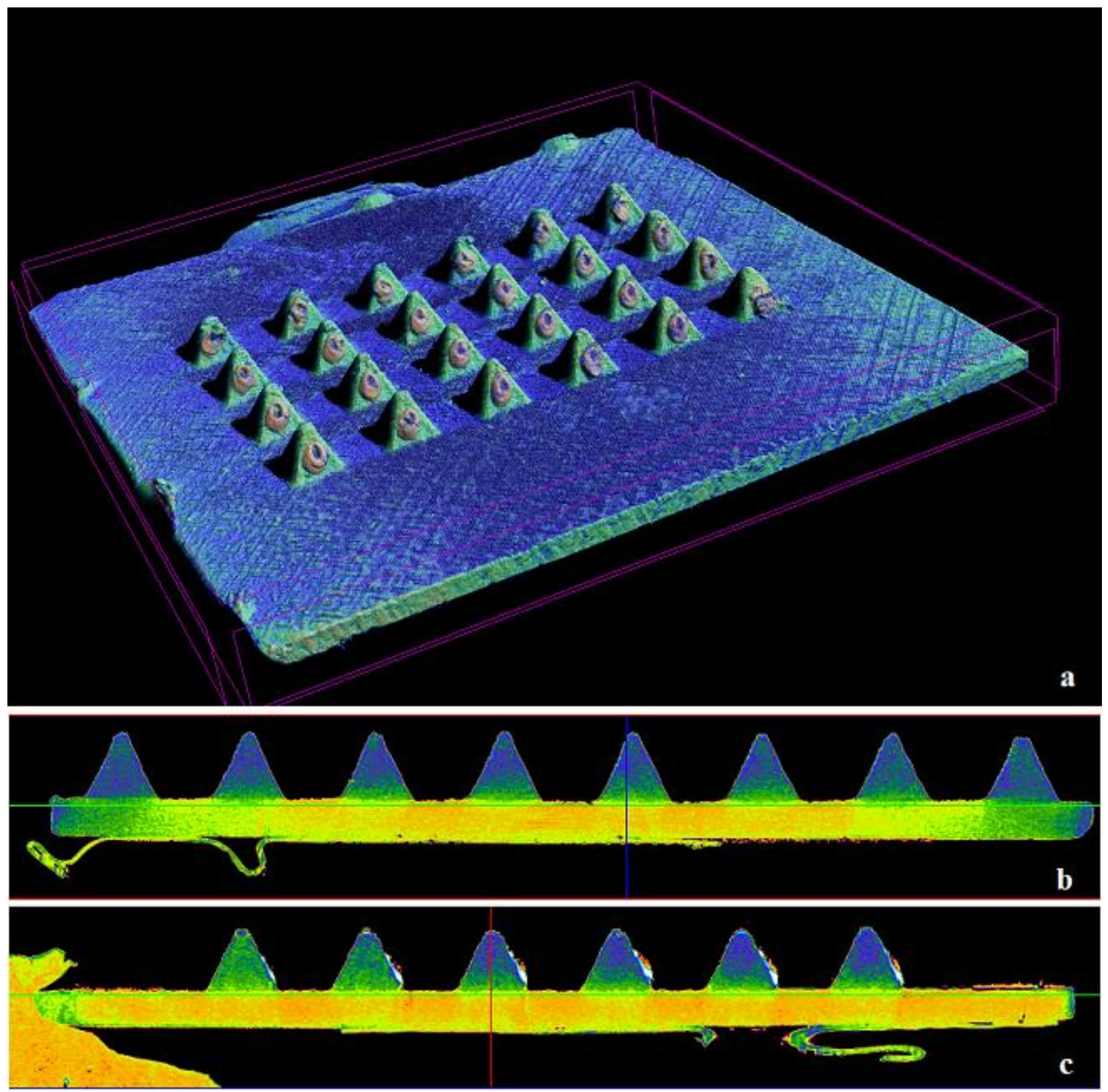

511 Figure 2. $\mu \mathrm{CT}$ images of the pyramid MN arrays coated with insulin:xylitol formulation.

512 (a) Overview; (b) image taken from the back side; (c) image taken from the left-hand side of the

513 array, showing the thin film coating. 

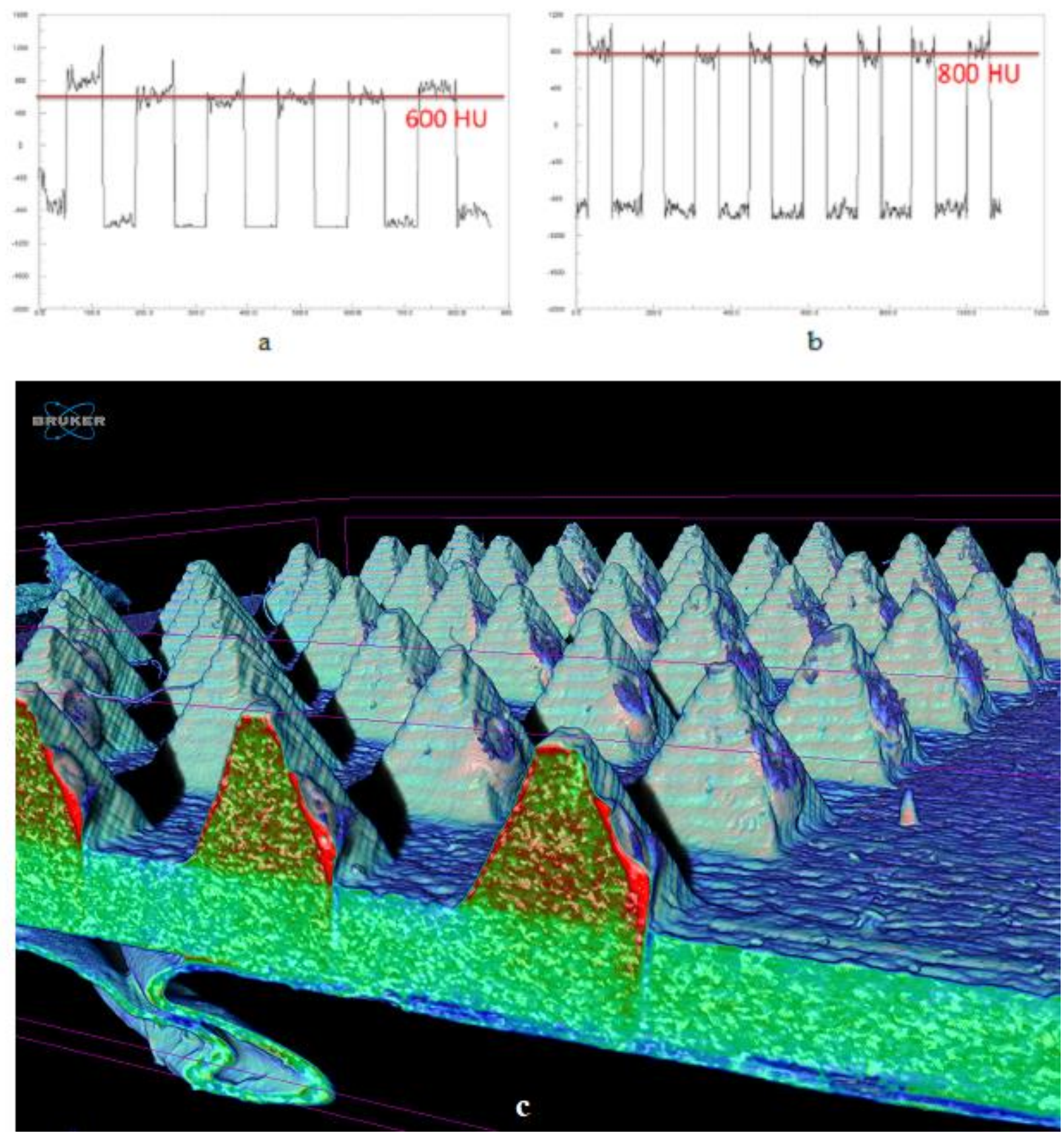

Figure 3. $\mu \mathrm{CT}$ evaluation. (a) Profile lines, measured across a single row of the control sample

516 (uncoated array) and (b) the coated pyramid MN array with insulin:xylitol formulation, showing

517 a $200 \mathrm{HU}$ increase; (c) cross-section of the coated MN array showing a fringe layer of 10-15 $\mu \mathrm{m}$. 


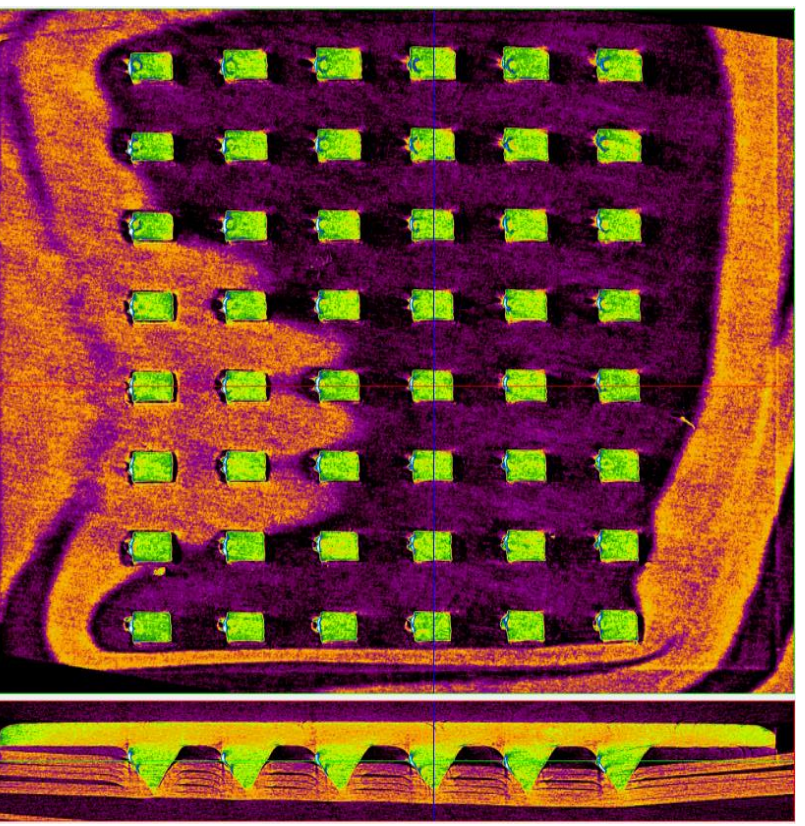

519 Figure 4. Cross section of $\mathrm{MN}$ array penetration through 8-ply strip of parafilm, applying a $5 \mathrm{~N}$ 520 load.

521 

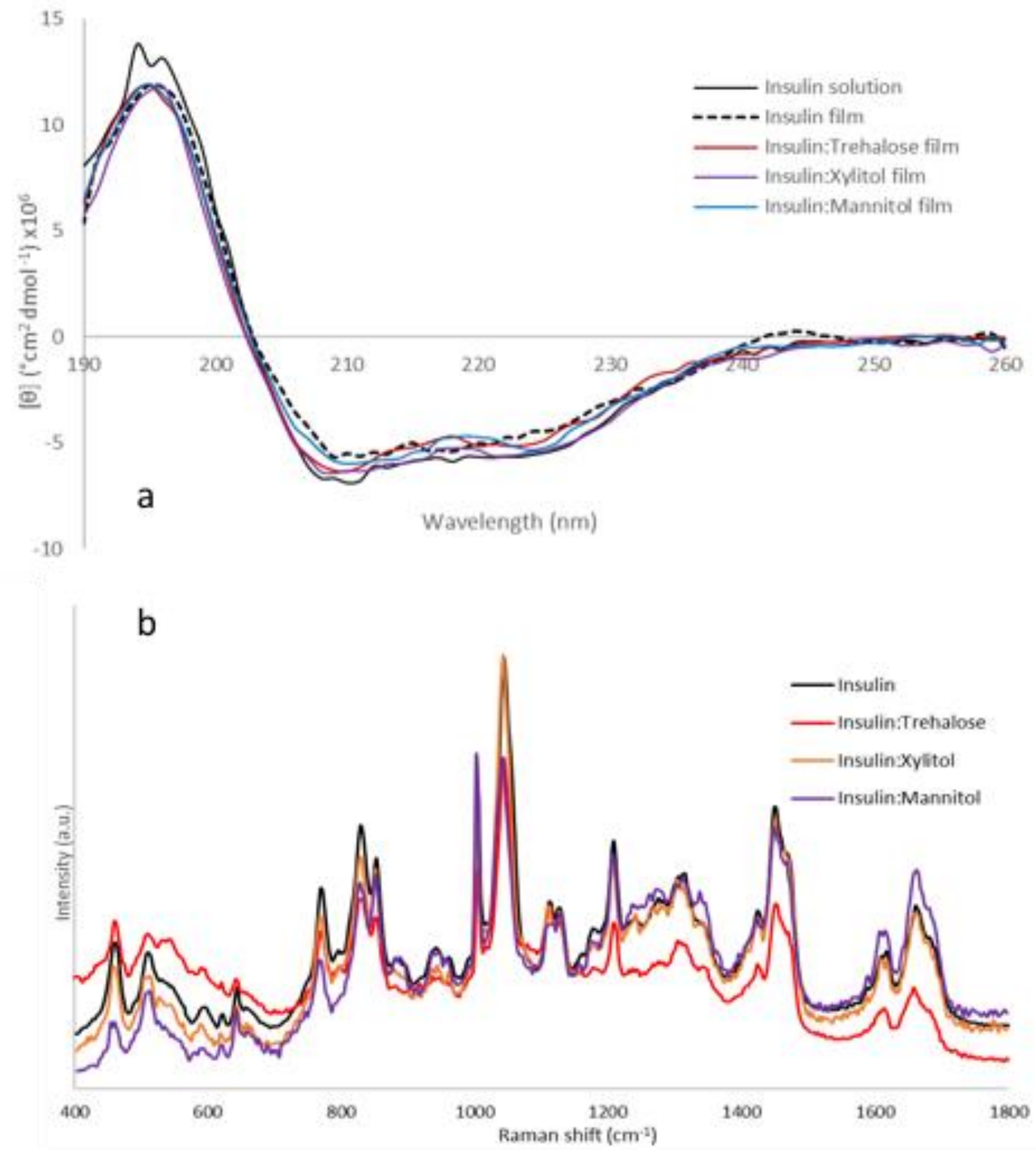

525 Figure 5. a) $\mathrm{CD}$ of insulin and insulin formulations and b) Raman spectra from 500 to $1800 \mathrm{~cm}^{-1}$ of pure insulin and insulin-sugars. 


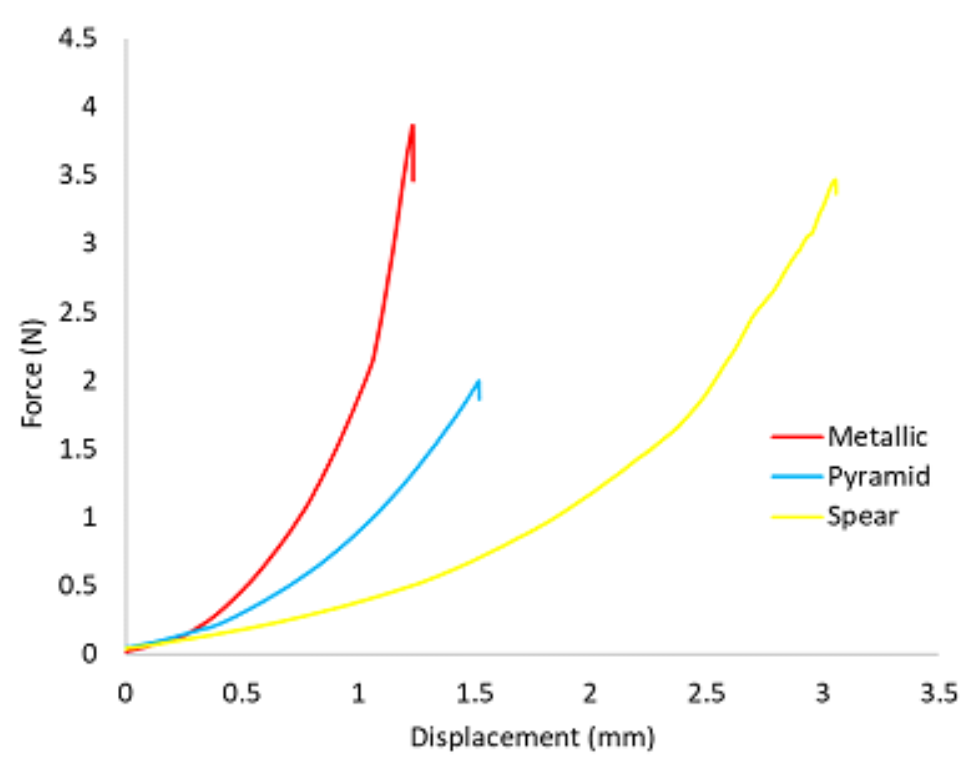

a

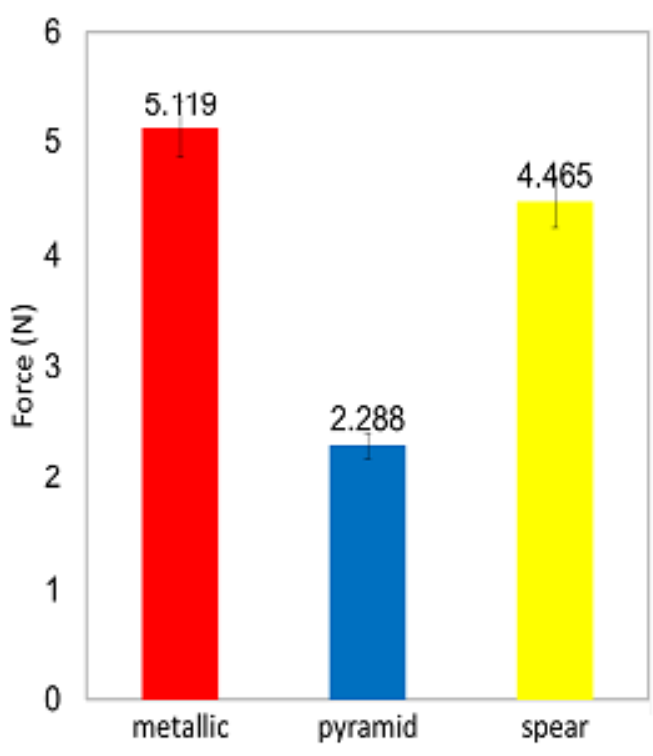

b

528 Figure 6. Penetration studies of MNs through porcine skin, comparing 3D printed spear and

529 pyramid designs with metallic MNs. (a) Force against displacement curves recorded during MN 530 insertion tests; (b) Maximum force required for MN penetration.

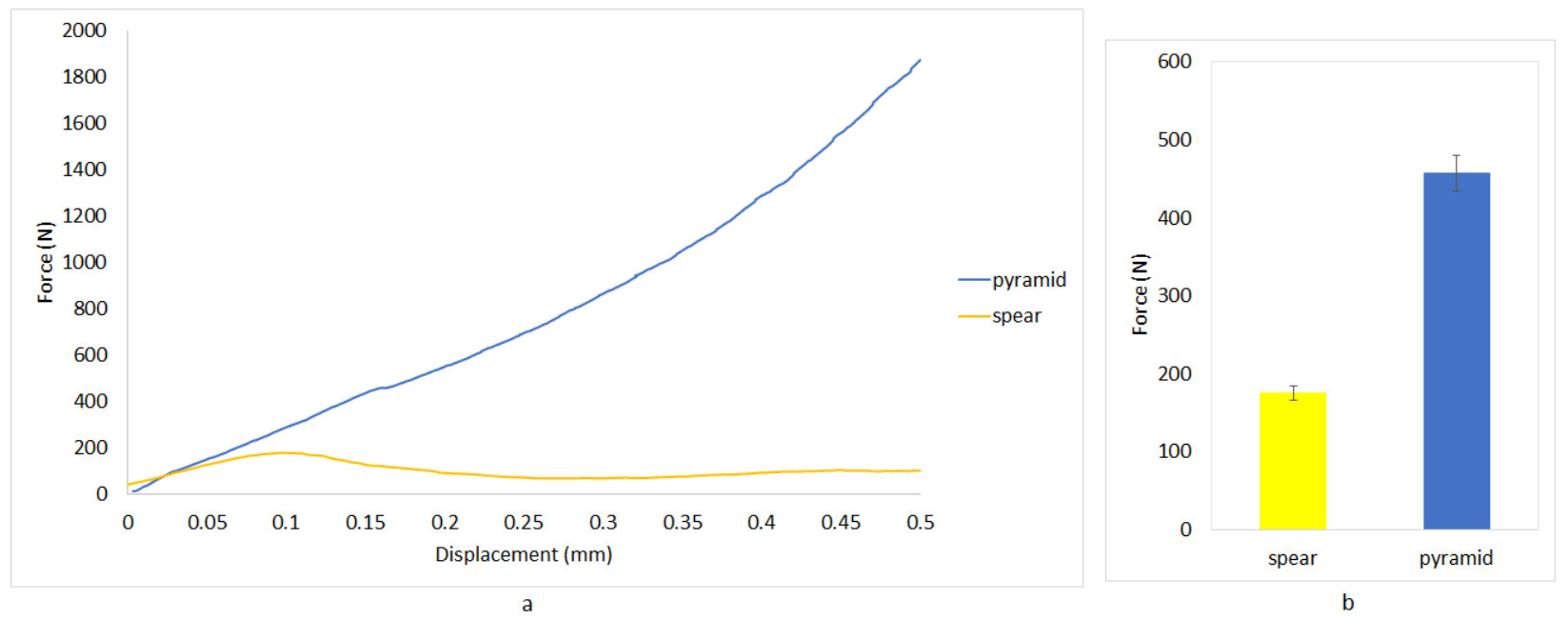

532 Figure 7. MN fracture testing for pyramid and spear designs. (a) Force against displacement 533 curves recorded during MN fracture tests; (b) Fracture MN strength 


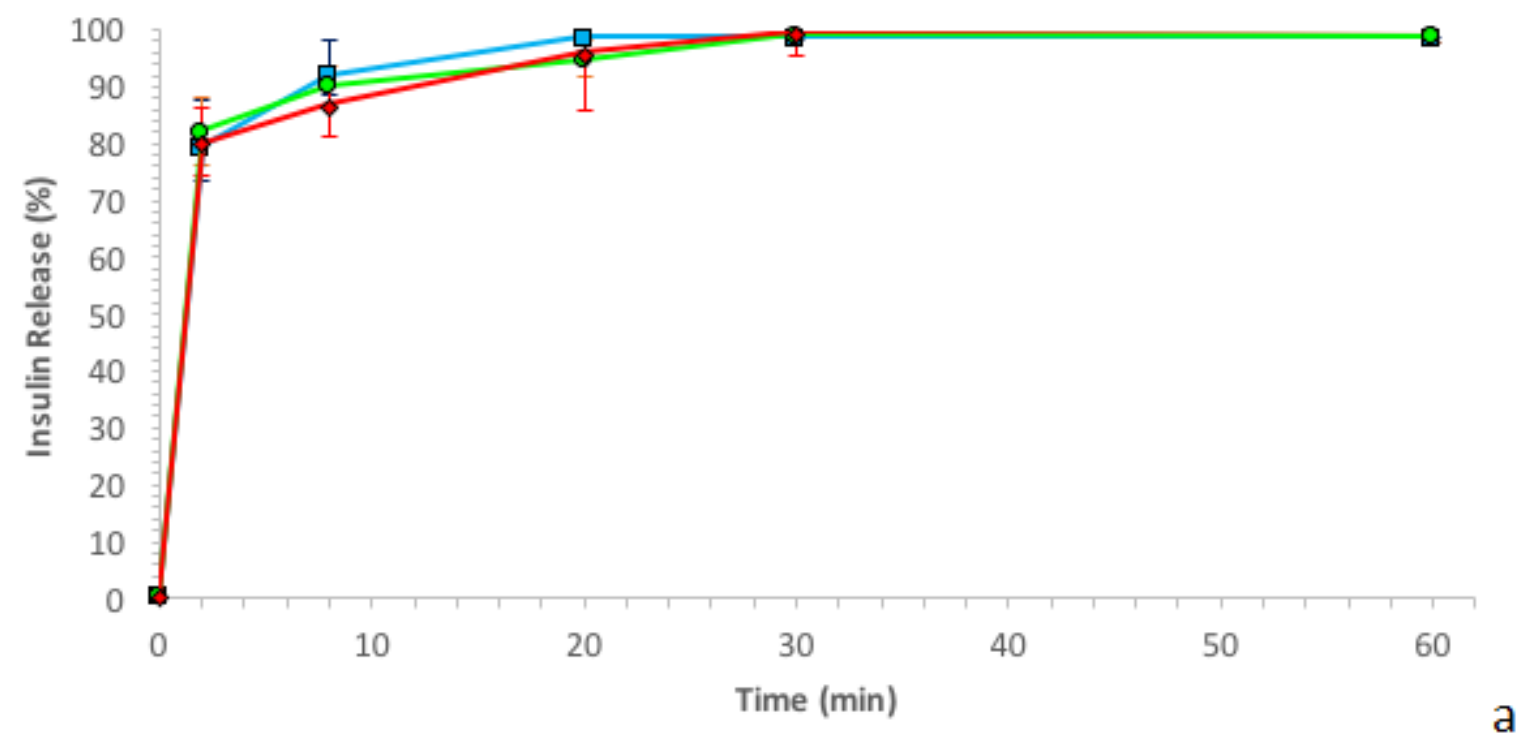

a

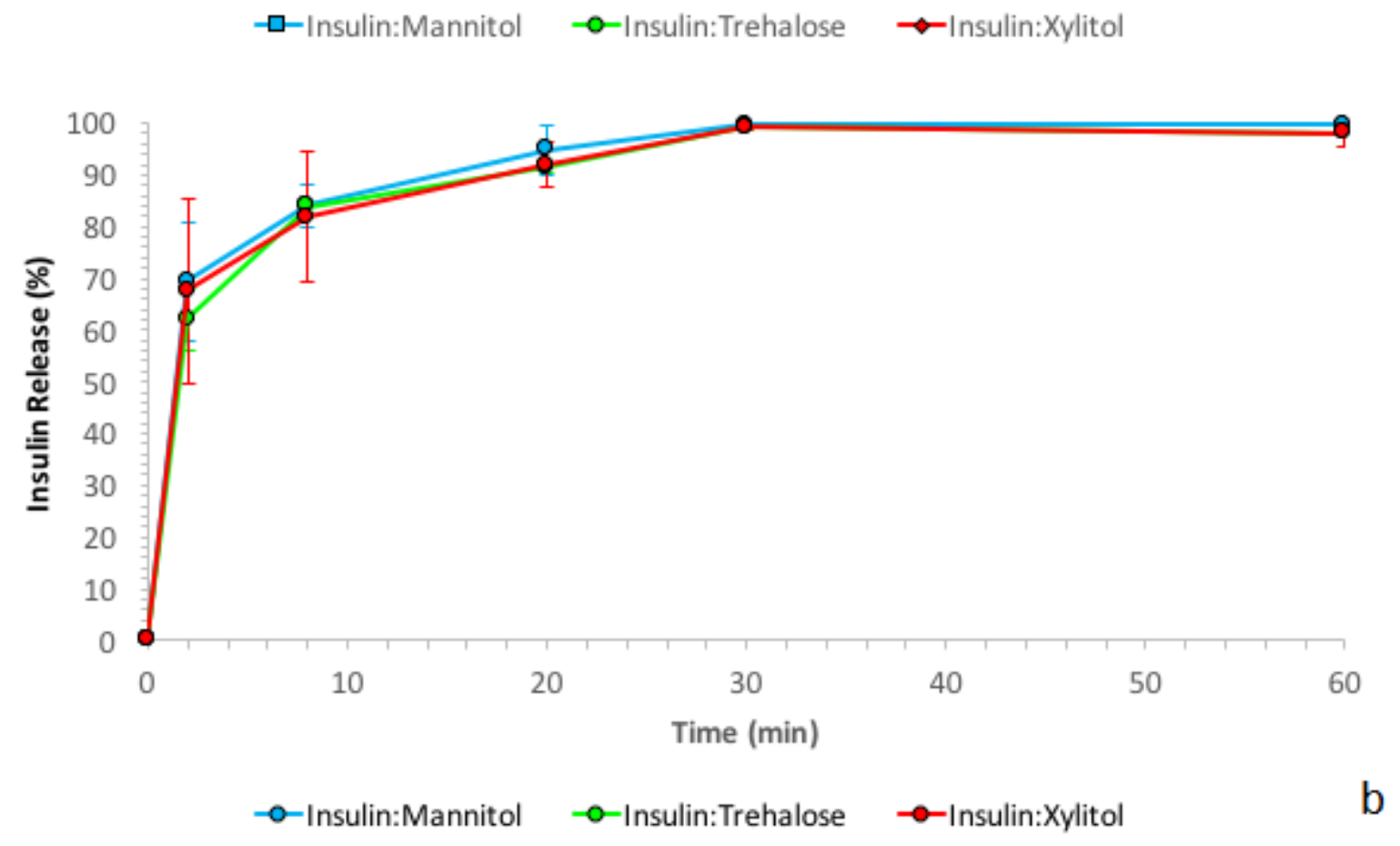

535

536 Figure 8. In vitro insulin release through porcine skin from a) the pyramid and b) the spear MN 537 designs for all investigated drug carriers. 


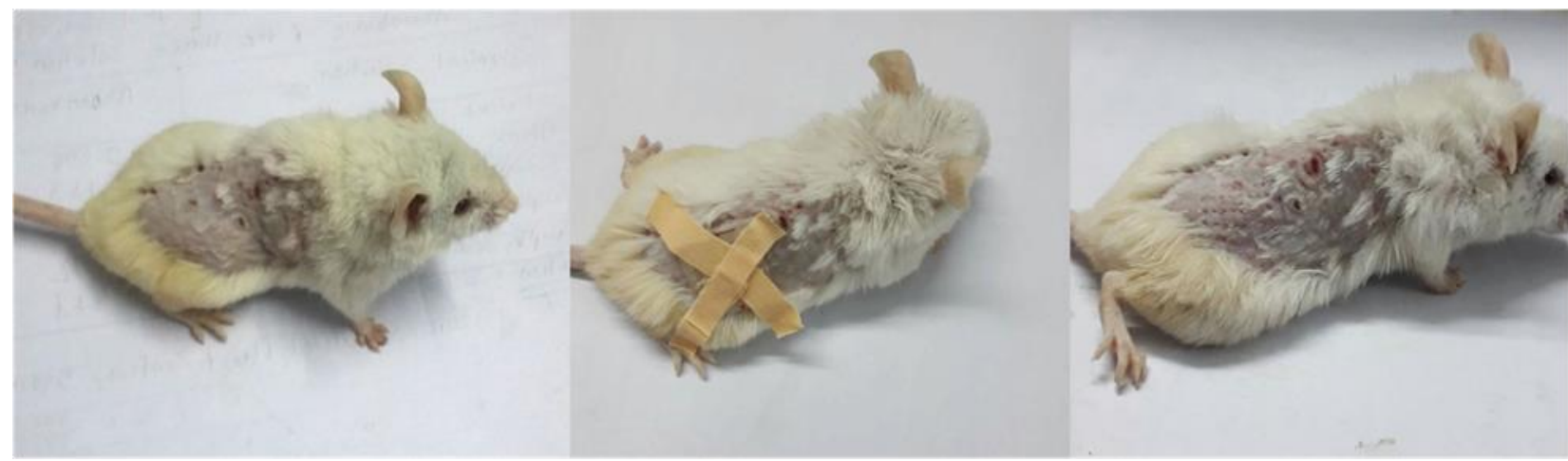

540 Figure 9. Experimental mice (A) before the application, (B) during the application and (C) after 541 removal of the 3D printed $\mathrm{MN}$ array for the delivery of insulin to diabetic mice. 

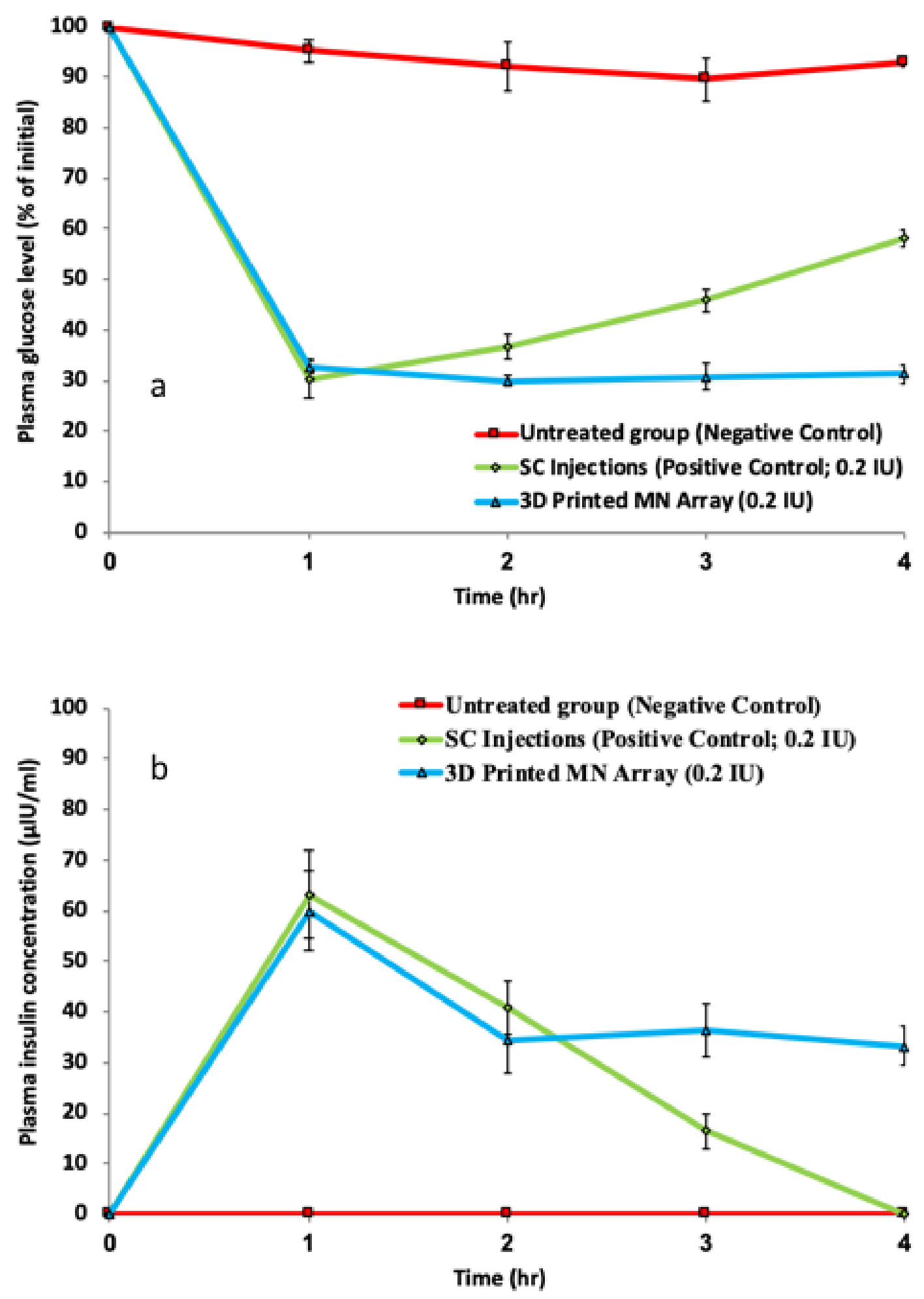

Figure 10. a) Comparative plasma glucose level vs time for untreated group, subcutaneous (SC) 544 injection and insulin-coated 3D Printed MN array applied to diabetic mice, over 4 hours $(n=3)$, 
545 b) comparative plasma insulin concentration vs time for untreated group, subcutaneous (SC)

546 injection and insulin-coated 3D Printed MN array applied to diabetic mice, over 4 hours $(n=3)$.

548 TABLES

549 Table 1 Treatment protocol for insulin-coated 3D printed MN array.

\begin{tabular}{|c|c|c|}
\hline Day & Stage & Treatment strategy \\
\hline-7 & $\begin{array}{l}\text { Animal model selection } \\
\text { and isolation }\end{array}$ & Transfer mice to study isolator \\
\hline 0 & Induction of diabetes & $\begin{array}{l}\text { - Weigh animals and injected with streptozotocin } \\
\text { (diabetes inducer) } \\
\text { - Daily blood glucose measurements }\end{array}$ \\
\hline 1 & & \\
\hline 2 & & \\
\hline 3 & Observation & Blood glucose level measurements \\
\hline 4 & & \\
\hline 5 & & \\
\hline 6 & & \\
\hline 7 & $\begin{array}{l}\text { Confirmation of } \\
\text { diabetes induction }\end{array}$ & Blood glucose level exceeds $300 \mathrm{mg} / \mathrm{dl}$ within 7 days. \\
\hline 8 & $\begin{array}{l}\text { Antidiabetic therapy } \\
\text { using insulin-coated 3D } \\
\text { printed MN array }\end{array}$ & $\begin{array}{l}\text { After treatment, hourly (up to } 4 \text { hours) blood samples } \\
\text { were collected from the jugular vein and the blood } \\
\text { glucose level was measured. }\end{array}$ \\
\hline & & The study was conducted for 24 hours. \\
\hline
\end{tabular}


Table 2. Pharmacodynamic parameters for plasma glucose levels of diabetic mice for untreated groups, subcutaneous (SC) injection (insulin dose: 0.2 IU) and 3D Printed MN array (insulin dose: $0.2 \mathrm{IU})(\mathrm{n}=3)$.

\begin{tabular}{ccccc}
\hline Group & $\mathbf{C}_{\min }(\boldsymbol{\%})$ & $\mathbf{T}_{\min }(\mathbf{h})$ & $\mathbf{A A C}_{\text {o to }}(\boldsymbol{\%} \mathbf{h r})$ & RPA (\%) \\
\hline Untreated groups & $89.5 \pm 4.2$ & 3 & $22.7 \pm 1.2$ & - \\
SC injections & $32.8 \pm 3.7$ & 1 & $208.5 \pm 3.7$ & 100 \\
3D Printed MN arrays & $30.1 \pm 1.0$ & 1 & $240.6 \pm 2.9$ & 122
\end{tabular}
the plasma glucose concentration vs. time curve; RPA, relative pharmacological availability compared to subcutaneous injection.

Table 3. Pharmacokinetic parameters for plasma glucose levels of diabetic mice for untreated 560 groups, subcutaneous (SC) injection (insulin dose: $0.2 \mathrm{IU}$ ) and 3D Printed MN array (insulin 561 dose: 0.2 IU) $(n=3)$.

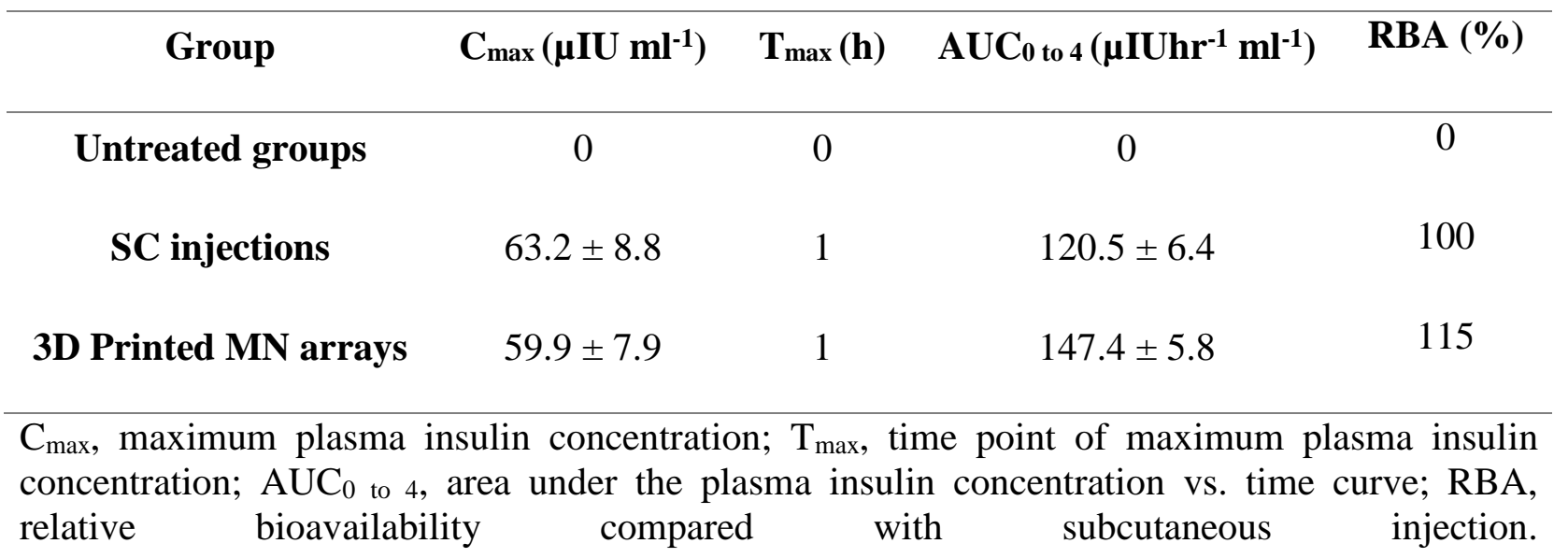


AUTHOR INFORMATION

\section{Corresponding Authors}

568 * Corresponding Authors: Prof. D.A. Lamprou, E-mail address: d.lamprou@qub.ac.uk, Tel.:

$569+44(0) 289097$ 2617. Prof. D. Douroumis, E-mail: d.douroumis@gre.ac.uk, Tel: +44 (0) 2083

$570 \quad 318440$.

\section{$571 \quad$ Author Contributions}

572 The manuscript was written through contributions of all authors. All authors have given approval 573 to the final version of the manuscript.

\section{$574 \quad$ Funding Sources}

575 This work was supported by the Coordenação de Aperfeiçoamento de Pessoal de Nível Superior

576 (CAPES) Foundation - Ministry of Education of Brazil; and the European Research Council 577 under the European Union's Seventh Framework Programme (FP/2007-2013) / ERC (grant 578 number. 615030).

\section{ABBREVIATIONS}

580 Transdermal Drug Delivery, TDD; MN, microneedle; poly(vinyl alcohol), PVA; glucose 581 oxidase, GOx; Additive Manufacturing, AM; UV, ultraviolet; CD, Circular Dichroism; $\mu \mathrm{CT}$, 582 Micro Computer Tomography; Scanning electron microscopy, (SEM); High-Performance Liquid 583 Chromatography, (HPLC); relative humidity, (RH).

585 The data will be available on request. 


\section{REFERENCES}

[1] A.Z. Alkilani, M.T.C. McCrudden, R.F. Donnelly, Transdermal drug delivery: Innovative pharmaceutical developments based on disruption of the barrier properties of the stratum corneum, Pharmaceutics. 7 (2015) 438-470. doi:10.3390/pharmaceutics7040438.

593 [2] W. Chen, H. Li, D. Shi, Z. Liu, W. Yuan, Microneedles As a Delivery System for Gene 594 Therapy, Front. Pharmacolocy. 7 (2016) 137. doi:10.3389/fphar.2016.00137.

595 [3] S.T. Sanjay, W. Zhou, M. Dou, H. Tavakoli, L. Ma, F. Xu, X.J. Li, Recent advances of controlled drug delivery using microfluidic platforms, Adv. Drug Deliv. Rev. 128 (2018) 3-28. doi:10.1016/j.addr.2017.09.013.

L. Goodchild, Could dissolvable microneedles replace injected vaccines?, Mater. Today. 18 (2015) 419-420. doi:10.1016/j.mattod.2015.08.005. Prausnitz, Lack of pain associated with microfabricated microneedles, Anesth. Analg. 92 (2001) 502-504. doi:10.1213/00000539-200102000-00041.

[6] D.P. Wermeling, S.L. Banks, D.A. Hudson, H.S. Gill, J. Gupta, M.R. Prausnitz, A.L. Stinchcomb, Microneedles permit transdermal delivery of a skin-impermeant medication to humans, Proc. Natl. Acad. Sci. 105 (2008) 2058-2063. doi:10.1073/pnas.0710355105. 4879-4890. doi:10.1002/adfm.201200864. 
$610[8]$ M. Korytkowski, L. Niskanen, T. Asakura, FlexPen®: Addressing issues of confidence

611

612

613

614

615

616

617

618

619

620

621

622

623

624

625

626

627

628

629

630

631

632 andconvenience in insulin delivery, Clin. Ther. 27 (2005) S89-100. doi:10.1016/j.clinthera.2005.11.019.

[9] X. Guo, W. Wang, Challenges and recent advances in the subcutaneous delivery of insulin, Expert Opin. Drug Deliv. $14 \quad$ (2017) 727-734. doi:10.1080/17425247.2016.1232247.

[10] R.J. Narayan, Transdermal delivery of insulin via microneedles, J. Biomed. Nanotechnol. 10 (2014) 2244-2260. doi:10.1166/jbn.2014.1976.

[11] J. Wang, Y. Ye, J. Yu, A.R. Kahkoska, X. Zhang, C. Wang, W. Sun, R.D. Corder, Z. Chen, S.A. Khan, J.B. Buse, Z. Gu, Core-Shell Microneedle Gel for Self-Regulated Insulin Delivery, ACS Nano. 12 (2018) 2466-2473. doi:10.1021/acsnano.7b08152.

[12] W. Yu, G. Jiang, Y. Zhang, D. Liu, B. Xu, J. Zhou, Polymer microneedles fabricated from alginate and hyaluronate for transdermal delivery of insulin, Mater. Sci. Eng. C. 80 (2017) 187-196. doi:10.1016/j.msec.2017.05.143.

[13] Y. Zhang, G. Jiang, W. Yu, D. Liu, B. Xu, Microneedles fabricated from alginate and maltose for transdermal delivery of insulin on diabetic rats, Mater. Sci. Eng. C. 85 (2018) 18-26. doi:https://doi.org/10.1016/j.msec.2017.12.006.

[14] S. Ross, N. Scoutaris, D. Lamprou, D. Mallinson, D. Douroumis, Inkjet printing of insulin microneedles for transdermal delivery, Drug Deliv. Transl. Res. 5 (2015) 451-461. doi:10.1007/s13346-015-0251-1.

[15] R.D. Pedde, B. Mirani, A. Navaei, T. Styan, S. Wong, M. Mehrali, A. Thakur, N.K. Mohtaram, A. Bayati, A. Dolatshahi-Pirouz, M. Nikkhah, S.M. Willerth, M. Akbari, Emerging Biofabrication Strategies for Engineering Complex Tissue Constructs, Adv. 
Mater. 29 (2017) 1-27. doi:10.1002/adma.201606061.

634 [16] L.E. Visscher, H.P. Dang, M.A. Knackstedt, D.W. Hutmacher, P.A. Tran, 3D printed

[19] S.N. Economidou, D.A. Lamprou, D. Douroumis, 3D printing applications for transdermal drug delivery, Int. J. Pharm. 544 (2018) 415-424. doi:10.1016/j.ijpharm.2018.01.031.

[20] B. Thavornyutikarn, P. Tesavibul, K. Sitthiseripratip, N. Chatarapanich, B. Feltis, P.F.A. Wright, T.W. Turney, Porous 45S5 Bioglass ${ }^{\circledR}$-based scaffolds using stereolithography: Effect of partial pre-sintering on structural and mechanical properties of scaffolds, Mater. Sci. Eng. C. 75 (2017) 1281-1288. doi:10.1016/j.msec.2017.03.001.

[21] D. Pede, G. Serra, D. De Rossi, Microfabrication of conducting polymer devices by inkjet stereolithography, Mater. Sci. Eng. C. 5 (1998) 289-291. doi:10.1016/S09284931(97)00056-8.

[22] E.J. Mott, M. Busso, X. Luo, C. Dolder, M.O. Wang, J.P. Fisher, D. Dean, Digital micromirror device (DMD)-based 3D printing of poly(propylene fumarate) scaffolds, Mater. Sci. Eng. C. 61 (2016) 301-311. doi:10.1016/j.msec.2015.11.071. 
656 [23] S.D. Gittard, P.R. Miller, C. Jin, T.N. Martin, R.D. Boehm, B.J. Chisholm, S.J. Stafslien, J.W. Daniels, N. Cilz, N.A. Monteiro-Riviere, A. Nasir, R.J. Narayan, Deposition of antimicrobial coatings on microstereolithography-fabricated microneedles, Jom. 63 (2011) 59-68. doi:10.1007/s11837-011-0093-3.

[24] Y. Lu, S.N. Mantha, D.C. Crowder, S. Chinchilla, K.N. Shah, Y.H. Yun, R.B. Wicker, J.W. Choi, Microstereolithography and characterization of poly(propylene fumarate)based drug-loaded microneedle arrays, Biofabrication. 7 (2015) 1-13. doi:10.1088/1758$5090 / 7 / 4 / 045001$.

[25] N. Sreerama, R.W. Woody, Estimation of protein secondary structure from circular dichroism spectra: Comparison of CONTIN, SELCON, and CDSSTR methods with an expanded reference set, Anal. Biochem. $287 \quad$ (2000) 252-260. doi:10.1006/abio.2000.4880.

[26] S.P. Davis, B.J. Landis, Z.H. Adams, M.G. Allen, M.R. Prausnitz, Insertion of microneedles into skin: Measurement and prediction of insertion force and needle fracture

[27] M.J. Uddin, N. Scoutaris, P. Klepetsanis, B. Chowdhry, M.R. Prausnitz, D. Douroumis, Inkjet printing of transdermal microneedles for the delivery of anticancer agents, Int. J. Pharm. 494 (2015) 593-602. doi:10.1016/j.ijpharm.2015.01.038.

[28] Z. Yong, D. Yingjie, W. Xueli, X. Jinghua, L. Zhengqiang, Conformational and bioactivity analysis of insulin: Freeze-drying TBA/water co-solvent system in the presence of surfactant and sugar, Int. J. Pharm. 371 (2009) 71-81. doi:10.1016/j.jpharm.2008.12.018.

678 [29] Y.H. Kim, C. Sioutas, K.S. Shing, Influence of stabilizers on the physicochemical 
characteristics of inhaled insulin powders produced by supercritical antisolvent process, Pharm. Res. 26 (2009) 61-71. doi:10.1007/s11095-008-9708-y.

[30] H. Schiffter, J. Condliffe, S. Vonhoff, Spray-freeze-drying of nanosuspensions: the manufacture of insulin particles for needle-free ballistic powder delivery, J. R. Soc. Interface. 7 (2010) S483-500. doi:10.1098/rsif.2010.0114.focus.

[31] L. Whitmore, B.A. Wallace, DICHROWEB, an online server for protein secondary structure analyses from circular dichroism spectroscopic data, Nucleic Acids Res. 32 (2004) 668-673. doi:10.1093/nar/gkh371.

[32] L. Whitmore, B.A. Wallace, Protein secondary structure analyses from circular dichroism spectroscopy: Methods and reference databases, Biopolymers. 89 (2008) 392-400. doi:10.1002/bip.20853.

[33] M.J. Ettinger, S.N. Timasheff, Optical activity of insulin. II. Effect of nonaqueous solvents, $\quad$ Biochemistry. $\quad 10 \quad$ (1971) 831-840. http://www.ncbi.nlm.nih.gov/entrez/query.fcgi?cmd=Retrieve \&db=PubMed\&dopt=Citati on\&list_uids=5544674.

[34] B. Sarmento, D.C. Ferreira, L. Jorgensen, M. van de Weert, Probing insulin's secondary structure after entrapment into alginate/chitosan nanoparticles, Eur. J. Pharm. Biopharm. 65 (2007) 10-17. doi:10.1016/j.ejpb.2006.09.005.

[35] F. Andrade, P. Fonte, M. Oliva, M. Videira, D. Ferreira, B. Sarmento, Solid state formulations composed by amphiphilic polymers for delivery of proteins: characterization and stability, Int. J. Pharm. 486 (2015) 195-206. doi:10.1016/j.ijpharm.2015.03.050.

[36] W. Dzwolak, R. Ravindra, J. Lendermann, R. Winter, Aggregation of bovine insulin probed by DSC/PPC calorimetry and FTIR spectroscopy, Biochemistry. 42 (2003) 11347- 
11355. doi:10.1021/bi034879h.

703

704

705

706

707

708

709

710

711

712

713

714

715

716

717

718

719

720

721

722

723

724

[37] J.F. Carpenter, J.H. Crowe, An infrared spectroscopic study of the interactions of carbohydrates with dried proteins, Biochemistry. 28 (1989) 3916-3922. doi:10.1021/bi00435a044.

[38] M.A. Haque, J. Chen, P. Aldred, B. Adhikari, Drying and denaturation characteristics of whey protein isolate in the presence of lactose and trehalose, Food Chem. 177 (2015) 816. doi:10.1016/j.foodchem.2014.12.064.

[39] S.G. Melberg, W.C. Johnson, Changes in secondary structure follow the dissociation of human insulin hexamers: A circular dichroism study, Proteins Struct. Funct. Bioinforma. 8 (1990) 280-286. doi:10.1002/prot.340080309.

[40] D.C.O. Nai-Teng Yu, C.S. Liu, Laser Raman Spectroscopy and the Conformation and Proinsulin of Insulin, J. Mol. Biol. 70 (1972) 117-132.

[41] P.O. Souillac, C.R. Middaugh, J.H. Rytting, Investigation of protein / carbohydrate interactions in the dried state . 2 . Diffuse reflectance FTIR studies, Int. J. Pharm. 235 (2002) 207-218.

[42] A. Das, P. Basak, R. Pattanayak, T. Kar, R. Majumder, D. Pal, A. Bhattacharya, M. Bhattacharyya, S.P. Banik, Trehalose induced structural modulation of Bovine Serum Albumin at ambient temperature, Int. J. Biol. Macromol. 105 (2017) 645-655. doi:10.1016/j.ijbiomac.2017.07.074.

[43] J. Lee, S. Timasheff, The Stabilization of Proteins by Sucrose *, J. Biol. Chem. 256 (1981) 7193-7201.

[44] S. Yoshioka, T. Miyazaki, Y. Aso, b-Relaxation of Insulin Molecule in Lyophilized Formulations Containing Trehalose or Dextran As a Determinant of Chemical Reactivity, 
Pharm. Res. 23 (2006) 961-966. doi:10.1007/s11095-006-9907-3.

726

727

728

729

730

731

732

733

734

735

736

737

738

739

740

741

742

743

744

745

746

747

[45] C. Branca, S. MacCarrone, S. Magazu, G. Maisano, S.M. Bennington, J. Taylor, Tetrahedral order in homologous disaccharide-water mixtures, J. Chem. Phys. 122 (2005) 174513-1-174513-6. doi:10.1063/1.1887167.

[46] N.K. Jain, I. Roy, Effect of trehalose on protein structure, Protein Sci. 18 (2009) 24-36. doi:10.1002/pro.3.

[47] G. Zeng, J.J. Shou, K.K. Li, Y.H. Zhang, In-situ confocal Raman observation of structural changes of insulin crystals in sequential dehydration process, Biochim. Biophys. Acta Proteins Proteomics. 1814 (2011) 1631-1640. doi:10.1016/j.bbapap.2011.09.002.

[48] L.G. Tensmeyer, J.E. Shields, E. Lilly, The Raman Spectra of Crystalline 4Zn, 2Zn, and Na Insulin., 1336 (1990) 222-234.

[49] H. Takeuchi, N. Watanabe, Y. Satoh, I. Harada, Effects of Hydrogen Bonding on the Tyrosine Raman Bands in the 1300-1150 cm - Region, 20 (1989) 233-237.

[50] S.D. Gittard, B. Chen, H. Xu, A. Ovsianikov, B.N. Chichkov, N.A. Monteiro-Riviere, R.J. Narayan, The effects of geometry on skin penetration and failure of polymer microneedles, J. Adhes. Sci. Technol. 27 (2013) 227-243. doi:10.1080/01694243.2012.705101.

[51] I.C. Lee, Y.C. Wu, S.W. Tsai, C.H. Chen, M.H. Wu, Fabrication of two-layer dissolving polyvinylpyrrolidone microneedles with different molecular weights for: In vivo insulin transdermal delivery, RSC Adv. 7 (2017) 5067-5075. doi:10.1039/c6ra27476e.

[52] J. Gupta, S.S. Park, B. Bondy, E.I. Felner, M.R. Prausnitz, Infusion pressure and pain during microneedle injection into skin of human subjects, Biomaterials. 32 (2011) 68236831. doi:10.1016/j.biomaterials.2011.05.061. 
748 [53] M. Ling, M. Chen, Dissolving polymer microneedle patches for rapid and efficient transdermal delivery of insulin to diabetic rats., Acta Biomater. 9 (2013) 8952-8961. doi:10.1016/j.actbio.2013.06.029.

751 [54] S. Liu, M. Jin, Y. Quan, F. Kamiyama, H. Katsumi, T. Sakane, A. Yamamoto, The 752 development and characteristics of novel microneedle arrays fabricated from hyaluronic acid, and their application in the transdermal delivery of insulin., J. Control. Release. 161 (2012) 933-41. doi:10.1016/j.jconrel.2012.05.030.

[55] S. Fakhraei Lahiji, Y. Jang, I. Huh, H. Yang, M. Jang, H. Jung, Exendin-4-encapsulated dissolving microneedle arrays for efficient treatment of type 2 diabetes, Sci. Rep. 8 (2018) 1-9. doi:10.1038/s41598-018-19789-x.

[56] Y. Qiu, G. Qin, S. Zhang, Y. Wu, B. Xu, Y. Gao, Novel lyophilized hydrogel patches for convenient and effective administration of microneedle-mediated insulin delivery, Int. J. Pharm. 437 (2012) 51-56. doi:10.1016/j.ijpharm.2012.07.035.

[57] S.P. Davis, W. Martanto, M.G. Allen, S. Member, M.R. Prausnitz, Hollow Metal Microneedles for Insulin Delivery to Diabetic Rats, 52 (2005) 909-915. 